\title{
Chaotic Multiobjective Evolutionary Algorithm Based on Decomposition for Test Task Scheduling Problem
}

\author{
Hui Lu, Lijuan Yin, Xiaoteng Wang, Mengmeng Zhang, and Kefei Mao \\ School of Electronic and Information Engineering, Beihang University, Beijing 100191, China \\ Correspondence should be addressed to Hui Lu; mluhui@buaa.edu.cn
}

Received 13 March 2014; Revised 20 June 2014; Accepted 20 June 2014; Published 15 July 2014

Academic Editor: Jyh-Hong Chou

Copyright ( 2014 Hui Lu et al. This is an open access article distributed under the Creative Commons Attribution License, which permits unrestricted use, distribution, and reproduction in any medium, provided the original work is properly cited.

\begin{abstract}
Test task scheduling problem (TTSP) is a complex optimization problem and has many local optima. In this paper, a hybrid chaotic multiobjective evolutionary algorithm based on decomposition (CMOEA/D) is presented to avoid becoming trapped in local optima and to obtain high quality solutions. First, we propose an improving integrated encoding scheme (IES) to increase the efficiency. Then ten chaotic maps are applied into the multiobjective evolutionary algorithm based on decomposition (MOEA/D) in three phases, that is, initial population and crossover and mutation operators. To identify a good approach for hybrid MOEA/D and chaos and indicate the effectiveness of the improving IES several experiments are performed. The Pareto front and the statistical results demonstrate that different chaotic maps in different phases have different effects for solving the TTSP especially the circle map and ICMIC map. The similarity degree of distribution between chaotic maps and the problem is a very essential factor for the application of chaotic maps. In addition, the experiments of comparisons of CMOEA/D and variable neighborhood MOEA/D (VNM) indicate that our algorithm has the best performance in solving the TTSP.
\end{abstract}

\section{Introduction}

Test task scheduling problem (TTSP) is an essential part of the automatic test system for improving throughput, reducing time, and optimizing resource allocation. Similar to other scheduling problems, the TTSP is one kind of combination optimization problems. It is illustrated to be an NP-hard problem through the analysis of the nature of the problem carried out by many researchers [1-3]. In addition, through the fitness distance analysis [4], we know that the TTSP has many local optima. The algorithm that has strong space searching ability is needed to solve the TTSP.

Recently, many intelligent methods are used for solving the TTSP and other similar scheduling problems based on the problems' character. All these kinds of researches focus on improving the searching ability of the algorithm and obtaining optimal or near-optimal solutions for the scheduling problem. There are two basic strategies. One is to propose an improvement algorithm based on the original algorithm, such as variable neighborhood multiobjective optimization algorithm based on decomposition (VNM) for the multiobjective test task scheduling problem [5]. Another is to adopt a hybrid algorithm using two different kinds of algorithms. For example, Lu et al. proposed a hybrid particle swarm optimization and taboo search strategies for the single objective TTSP [6]. Recently, the hybrid method becomes a mainstream.

Different from the hybrid method using different kinds of algorithms, using chaos in the evolutionary process represents its advantages in improving the searching ability. $\mathrm{Lu}$ et al. proposed a chaotic nondominated sorting genetic algorithm for the multiobjective test task scheduling problem and validated the best performance in convergence and diversity through the experiment and analysis [1]. Donald et al. utilized the chaos-induced discrete self-organizing migrating algorithm to solve the lot-streaming flow shop scheduling problem with setup time [7]. Gavrilova and Ahmadian studied an on-demand chaotic neural network for the broadcast scheduling problem and found an optimal time division multiple access (TDMA) frame [8]. Jiang et al. proposed a chaos-based fuzzy regression approach to model customer satisfaction for product design and used a chaotic optimization algorithm to generate the polynomial structures 
of customer satisfaction models [9]. Sun et al. studied a novel hysteretic noisy chaotic neural network for broadcast scheduling problems in packet radio networks and exhibited a stochastic chaotic simulated annealing algorithm [10]. The phenomenon that occurred in these researches illustrated that the evolutionary algorithm embedded with chaos is an effective and efficient approach for improving the searching ability of the algorithm. However, all these researches have the same defect. The authors only used one or several chaotic maps embedded in algorithms to solve practical problems. However, there is no detailed discussion and analysis.

For the TTSP, notice that the previous studies mostly aim at a single objective and only few papers focus on the multiobjective problem $[1,5]$. Through the analysis of our previous related work for the multiobjective TTSP, we know the multiobjective evolutionary algorithm based on decomposition (MOEA/D) exhibited the best performance in solving the TTSP [5] in the aspect of convergence and diversity. Therefore, using chaotic maps in MOEA/D can further enhance the quality of the solution for the TTSP.

In this paper, we propose a chaotic multiobjective evolutionary algorithm based on decomposition (CMOEA/D) for the TTSP. Ten chaotic maps are embedded in three different phases in the evolutionary process. The aim is to give guidance for the choice of chaotic maps and phases based on the framework of MOEA/D for the TTSP.

First, the chromosome-encoding scheme is very important for the problem description and the operation in the evolutionary process. For the TTSP, there are different kinds of encoding strategies, like task sequencing list (TSL) (or the operations list coding (OLC)), matrix-encoding, and integrated encoding scheme (IES). TSL and matrix-encoding are not acceptable if a task can be tested on more than one set of instruments. IES can overcome this problem, but it cannot realize the selection operation under equal probability. Therefore, in this paper, the improved IES is proposed by changing the schemes selection method of every test task. As a result, the equal probability is realized.

Then, ten chaotic maps are embedded in MOEA/D independently in three phases. The ten chaotic maps are baker's map, cat map, circle map, cubic map, Gauss map, ICMIC map, logistic map, sinusoidal map, tent map, and Zaslavskii map. Three phases are initial population, crossover operator, and mutation operator. Four benchmarks of the TTSP are used to evaluate the performance of the proposed algorithm. They are $6 \times 8,20 \times 8,30 \times 12$, and $40 \times 12$. We use $n \times m$ to represent the benchmark. Here, $n$ is the number of tasks and $m$ is the number of instruments.

The performance metrics hypervolume (HV) and $C$ [11] are used to evaluate the role of chaotic maps on MOEA/D. Therefore, we can find which kind of chaotic map embedded algorithm is the best one for solving the TTSP.

From the results of experiments, it can be seen that the chaotic map embedded MOEA/D has good performance to solve the TTSP for both small and large scale problems. Different kinds of chaotic maps have different performances in different phases of MOEA/D, but ICMIC map and circle map in initial population, crossover operator, and mutation operator have the best performance. The experiments for comparisons of CMOEA/D and VNM show that our algorithm performs better than the VNM in solving the TTSP. The evidence, the chaotic map is an effective and efficient method for solving the problem with local optima, is validated. The similarity degree of distribution between chaotic maps and the problem is a very essential factor for the application of chaotic maps.

The rest of the paper is organized as follows. Section 2 gives a summary of related work on applying chaos to improve evolutionary algorithms. Section 3 concludes the mathematical model proposed by us in previous work for the integrity. In Section 4, ten chaotic maps including both one-dimensional maps and two-dimensional maps are introduced. In Section 5, the proposed CMOEA/D is described in detail for solving the TTSP. The detail of the encoding scheme and the phases in which chaos can be embedded in evolutionary algorithms are introduced. Experimental results and performance comparisons are presented and discussed in Section 6. Finally, Section 7 concludes the paper.

\section{Related Work}

Recently, chaotic sequences have been integrated in the evolutionary process through two types of operations. One is using chaotic maps to replace random sequences. Another is to replace the genetic operations. These two kinds of operations always appear in the same algorithm at once.

In detail, all the operations can be divided into seven cases. They are population initialization, setting crossover probability, setting crossover position, setting crossover operator, setting mutation probability, setting mutation operator, and increasing chaotic disturbance. The performance of different operations is totally different. For example, adopting chaotic maps in the initialization can maintain the population diversity. The aim of using chaotic maps to replace standard mutation operator is to avoid the search being trapped in local optima.

For the scheduling problem, the situation is the same as the above in both the single objective and the multiobjective scheduling problems. For the single objective scheduling problem, Cheng et al. used the hybrid genetic algorithm and chaos to optimize the hydropower reservoir operation [12]. Two methods were adopted to improve the performance of GA. One was the adoption of chaos for initialization, and another was the annealing chaotic mutation operation. The conclusion was that the proposed approach is feasible and effective in optimal operations of complex reservoir systems. Liu and Cao [13] proposed a chaotic algorithm for the fuzzy job scheduling problem in the grid environment with uncertainties. The authors incorporated logistic map with the standard genetic algorithm and proposed a chaotic mutation operator based on the feedback of the fitness function. Singh and Mahapatra [14] proposed a swarm optimization approach for the flexible flow shop scheduling problem with multiprocessor tasks. The logistic map was used in this paper. Bahi et al. [15] considered a novel chaos-based scheduling scheme for video surveillance to defeat malicious intruders. The concept of chaotic iterations was investigated. 
$\mathrm{Yu}$ and $\mathrm{Gu}$ [16] proposed an improved transiently chaotic neural network approach for the identical parallel machine scheduling problem.

For the multiobjective scheduling problem, Niknam et al. [17] proposed an improved particle swarm optimization (IPSO) for the multiobjective optimal power flow problem considering the cost, loss, emission, and voltage stability index. To improve the quality of solutions, particularly to avoid being trapped in local optima, this study presented an IPSO that profits from chaos and self-adaptive concepts to adjust the particle swarm optimization parameters. Zhou et al. [18] established time, expenses, resources, and quality objective functions and used the chaos particle swarm optimization to solve the resource-constrained project scheduling problem. Fang [19] proposed a quantum immune algorithm for the multiobjective parallel machine scheduling problem in textile manufacturing industry. Here, a novel mutation operator with a chaos-based rotation gate was investigated. We proposed a chaotic nondominated sorting genetic algorithm (CNSGA) to solve the test task scheduling problem. According to the different capabilities of the logistic and the cat chaotic operators, the CNSGA approach using the cat population initialization, the cat or logistic crossover operator, and the logistic mutation operator has good performance [1].

All these researches, despite the single objective or the multiobjective problem in these scheduling fields, have the same features. The chaotic maps are used for improving the searching ability of the evolutionary algorithm. However, most of researches only used one kind of chaotic maps embedded in special phases of the algorithm, and comprehensive analysis is inefficient. In fact, different kinds of the scheduling problems have different characters, and different chaotic maps have different effects on the algorithms and the problems. Our work will focus on the analysis and design of chaotic multiobjective algorithm for the TTSP. We investigate the guidance for solving the TTSP.

\section{Mathematical Model for the TTSP}

3.1. The Problem Description. The aim of the TTSP is to organize the execution of $n$ tasks on $m$ instruments. In this problem, there are a set of tasks $T=\left\{t_{j}\right\}_{j=1}^{n}$ and a set of instruments $R=\left\{r_{i}\right\}_{i=1}^{m}$. The notifications $P_{j}^{i}, S_{j}^{i}$, and $C_{j}^{i}$ present the test time, the test start time, and the test completion time of task $t_{j}$ tested on $r_{i}$, respectively [1]. For the TTSP, one task must be tested on one or more instruments. In other words, some instruments collaborate for one test task. A variable $O_{j}^{i}$ is defined to express whether the task $t_{j}$ occupies the instrument $r_{i}$. A task $t_{j}$ could have several alternative schemes to complete the test. $W_{j}=\left\{w_{j}^{k}\right\}_{k=1}^{k_{j}}$ is used to denote the alternative schemes of task $t_{j}$, where $k_{j}$ is the number of schemes of $t_{j} . K=\left\{k_{j}\right\}_{j=1}^{n}$ is the set containing the numbers of schemes that correspond to every task. Each $w_{j}^{k}$ is a subset of $R$ and can be represented as $w_{j}^{k}=\left\{r_{j k}^{u}\right\}_{u=1}^{u_{j k}}$. Here, $u_{j k}$ is the number of instruments for $w_{j}^{k}$. Obviously, $\cup_{1 \leq k \leq k_{j}, 1 \leq j \leq n} w_{j}^{k}=R$. The notification $P_{j}^{k}=\max _{r_{i} \in w_{j}^{k}} P_{j}^{i}$ is used to express the test time of $t_{j}$ for $w_{j}^{k}$.

3.2. Constraint Relationship. The TTSP has two types of constraints: the restriction on resources and the precedence constraint between the tasks. The restriction on resources can be expressed as follows:

$$
X_{j j^{*}}^{k k^{*}}= \begin{cases}1 & \text { if } w_{j}^{k} \cap w_{j^{*}}^{k^{*}} \neq \varnothing, \\ 0 & \text { otherwise. }\end{cases}
$$

The precedence constraint between the tasks can be represented as follows:

$$
Y_{j j^{*}}= \begin{cases}0 & \text { if } t_{j} \text { and } t_{j^{*}} \text { have equal priorities, } \\ +d & \text { if } t_{j} \text { needs to be tested before } t_{j^{*}} \\ & \text { with at least } d \text { unit time, that is, } t_{j}>t_{j^{*}}, \\ -d \quad & \text { if } t_{j^{*}} \text { needs to be tested before } t_{j} \\ & \text { with at least } d \text { unit time, that is, } t_{j^{*}}>t_{j},\end{cases}
$$

where $d \in R^{+}$. In this paper, $d$ equals the test time of the high priority task.

3.3. Objective Function. In this study, we consider two objective functions. The model is defined as follows:

$$
\operatorname{minimize}\left\{\max _{\substack{1 \leq k \leq k_{j} \\ 1 \leq j \leq n}} \max _{i} \in w_{j}^{k} C_{j}^{i}, \frac{1}{Q} \sum_{j=1}^{n} \sum_{i=1}^{m} P_{j}^{i} O_{j}^{i}\right\},
$$

subject to

$$
\begin{gathered}
C_{j}^{i}=S_{j}^{i}+P_{j}^{i}, \\
O_{j}^{i}= \begin{cases}1 & \text { if } t_{j} \text { occupies } r_{i}, \\
0 & \text { otherwise. }\end{cases}
\end{gathered}
$$

The first objective function minimizes the maximal test completion time and the second objective function minimizes the mean workload of the instruments. Here, $Q$ denotes the parallel steps. The initial value of $Q$ is 1 . Assign the instruments for all of the tasks if $X_{j j^{*}}^{k k^{*}}=1, Q=Q+1$.

Constraint (4) indicates that the setup time of the instruments and the move time between the tasks are negligible. Constraint (5) defines whether the task $t_{j}$ occupies the instrument $r_{i}$. Here, we assume $P_{j}^{i}=P_{j}^{k}$ to simplify the problem.

\section{Chaotic Maps}

Ten chaotic maps including both one-dimensional maps and two-dimensional maps are introduced in this section. Each one has specific features, and different chaotic maps combined with optimization algorithms have different results (Table 1). 
TABLE 1: The list of chaotic maps.

\begin{tabular}{|c|c|c|c|}
\hline Chaotic map & Formula & Dimensions & Range \\
\hline Baker's map & $B(x, y)= \begin{cases}(2 x, 2 y) & \text { for } 0 \leq x<0.5 \\
\left(2-2 x, 1-\frac{y}{2}\right) & \text { for } 0.5 \leq x<1\end{cases}$ & 2 & $x \in(0,1)$ \\
\hline Arnold's cat map & $\begin{array}{l}x_{k+1}=x_{k}+y_{k} \bmod (1) \\
y_{k+1}=x_{k}+2 y_{k} \bmod (1)\end{array}$ & 2 & $x_{k} \in(0,1)$ \\
\hline Circle map & $\begin{array}{l}x_{k+1}=\left\{x_{k}+b-\left(\frac{a}{2 \pi}\right) \sin \left(2 \pi x_{k}\right)\right\} \bmod (1) \\
a=0.5 \quad b=0.2\end{array}$ & 1 & $(0,1)$ \\
\hline Cubic map & $\begin{array}{l}x_{k+1}=\rho x_{k}\left(1-x_{k}^{2}\right), \quad x_{k} \in(0,1) \\
\rho=2.59\end{array}$ & 1 & $(0,1)$ \\
\hline Gauss map & $x_{k+1}= \begin{cases}0 & x_{k}=0 \\
\frac{1}{x_{k}} \bmod (1) & \text { otherwise }\end{cases}$ & 1 & $(0,1)$ \\
\hline ICMIC map & $\begin{array}{l}x_{k+1}=\sin \left(\frac{a}{x_{k}}\right), \quad a \in(0, \infty), \quad x_{k} \in(-1,1) \\
a=2\end{array}$ & 1 & {$[-1,1]$} \\
\hline Logistic map & $\begin{array}{l}x_{k+1}=a x_{k}\left(1-x_{k}\right) \\
a=4\end{array}$ & 1 & $(0,1)$ \\
\hline Sinusoidal map & $x_{k+1}=\sin \left(\pi x_{k}\right), \quad x_{k} \in(0,1)$ & 1 & $(0,1)$ \\
\hline Tent map & $x_{k+1}= \begin{cases}\frac{x_{k}}{0.7} & x_{k}<0.7 \\
\left(\frac{10}{3}\right) x_{k}\left(1-x_{k}\right) & \text { otherwise }\end{cases}$ & 1 & $(0,1)$ \\
\hline Zaslavskii map & $\begin{array}{c}x_{k+1}=\left(x_{k}+v+a y_{k+1}\right) \bmod (1) \\
y_{k+1}=\cos \left(2 \pi x_{k}\right)+e^{-r} y_{k} \\
v=400 \quad r=3 \quad a=12.6695\end{array}$ & 2 & $y_{k} \in[-1.0512,1.0512]$ \\
\hline
\end{tabular}

There are two problems for these chaotic maps. One is that the range of ICMIC and Zaslavskii maps is not $(0,1)$. As a result, the generated chaotic sequences need the scale transformation. Another is some maps, like tent map, have fixed points. Therefore, jumping out from fixed points is necessary for maintaining the chaos characteristics.

Figure 1 shows the distribution of different chaotic maps. It reveals that baker's map, bat map, and tent map have uniform distribution, while other chaotic maps, like circle map, cubic map, ICMIC map, logistic map, and Zaslavskii map, have nonuniform distribution, relatively.

\section{Chaotic Multiobjective Evolutionary Algorithm Based on Decomposition}

5.1. The Improving Encoding Method for the TTSP. Integrated encoding scheme (IES) proposed by our previous research [1] can use one chromosome to contain the information about both the processing sequence of the tasks and the occupancy of the instruments for each task. It can transform a discrete optimization problem into a continuous optimization problem. Therefore, the encoding efficiency is improved, and the complexity of the genetic manipulations is reduced.

Here, we use an example with four tasks and four instruments for illustration of the role of IES. The detail is in Table 2.
The main concept of the IES is to use the relationships between the decision variables to express the sequence of tasks and use the values of the variables to represent the occupancy of the instruments for each task. This concept is illustrated in Table 3.

The entries in the first row are the decision variables, which range between 0 and 1 . They are sorted in ascending order. The rank of every variable denotes a test task index in the sequence. Thus, the second row (or the task sequence) is obtained. On the other hand, the instrument assignment can also be obtained from the decision variables. If we want to know which instruments will be occupied by the task $t_{j}, w_{j}^{k}$ should be ascertained. In other words, we should know the value of $k$, which can be calculated by the decision variable corresponding to $t_{j}$. The formula is as follows:

$$
k=\left[x_{i j} \times 10\right] \bmod k_{j}+1 .
$$

Here, $x_{i j} \in[0,1]$ represents the decision variable that corresponds to $t_{j}$, and $k_{j}$ is the number of schemes of $t_{j}$. For example, for the task $t_{1}$, the corresponding decision variable is 0.1270 , and the number of schemes is $k_{1}=2$. Then, the value of $k$ can be calculated as follows according to (6): $k=$ $[0.1270 \times 10] \bmod k_{1}+1=1 \bmod 2+1=2$. Therefore, $w_{1}^{2}=\left\{r_{2}, r_{4}\right\}$ is occupied.

However, this encoding scheme has one defect that all schemes are selected with unequal probability. For example, 

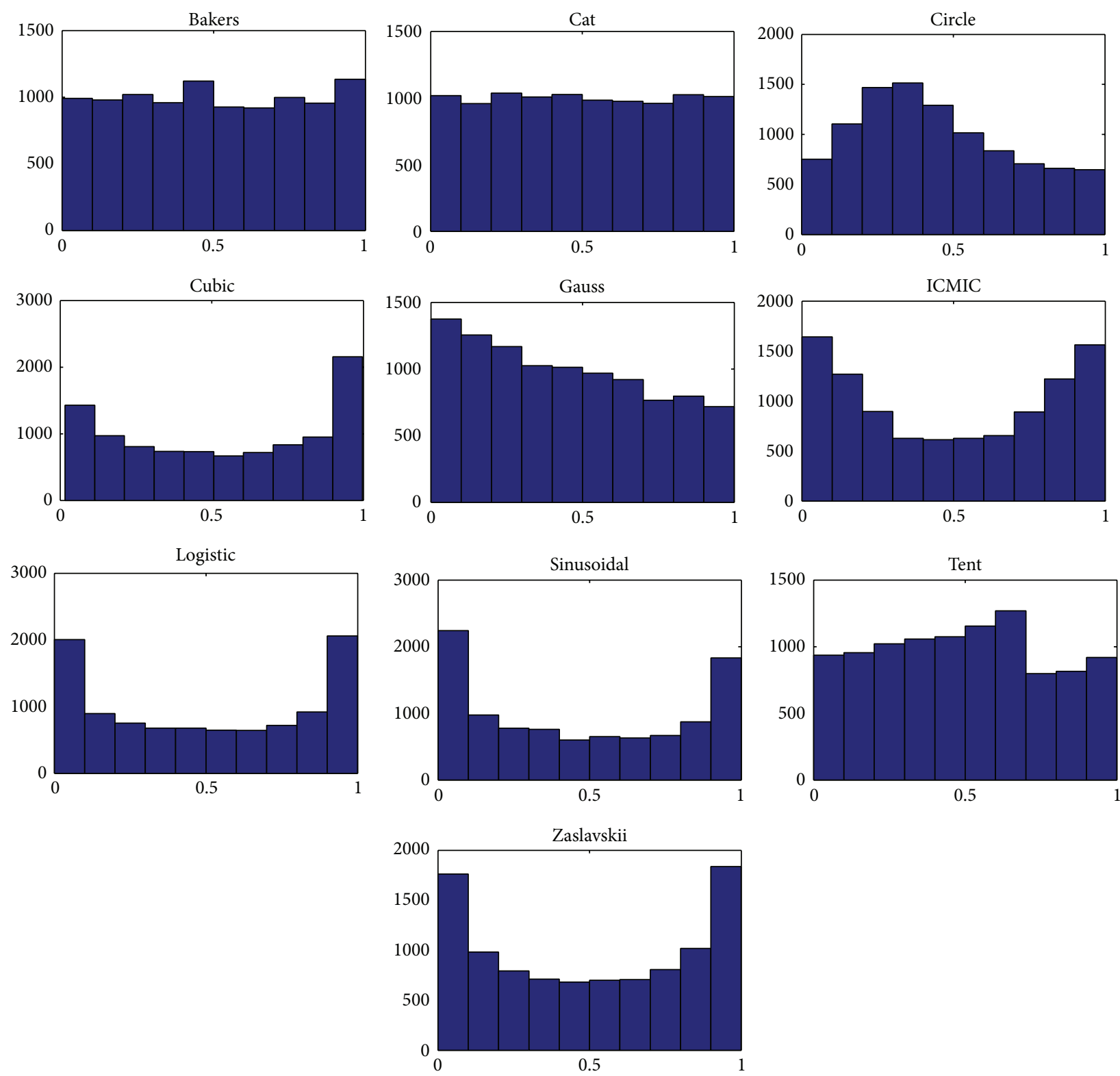

FiguRE 1: Distribution of different chaotic maps.

for one task $t_{4}$, the number of schemes is $k_{4}=3$. Then, the value of $k$ can be calculated according to (6) as shown in Table 4.

As seen from Table 4 , the probability of $k=1$ is $4 / 10$, but $3 / 10$ for $k=2,3$. It means all schemes are selected with unequal probability. Based on the original formula, we improved the encoding strategy as follows:

$$
k=\left[x_{i j} k_{j} \times 10\right] \bmod k_{j}+1 \text {. }
$$

Then, the value of $k$ can be calculated according to (7) as shown in Table 5.

As seen from Table 5, the equal probability of $k=1,2,3$ is $1 / 3$. This encoding method never generates duplication of a certain task and does not generate unfeasible solutions. In addition, equal probability can maintain impartiality for all
TABLE 2: A TTSP with four tasks and four instruments.

\begin{tabular}{lccc}
\hline$T$ & $W_{j}$ & $w_{j}^{k}$ & $P_{j}^{k}$ \\
\hline$t_{1}$ & $w_{1}^{1}$ & $r_{1}, r_{2}$ & 5 \\
& $w_{1}^{2}$ & $r_{2}, r_{4}$ & 3 \\
\hline \multirow{2}{*}{$t_{2}$} & $w_{2}^{1}$ & $r_{1}$ & 4 \\
& $w_{2}^{2}$ & $r_{3}$ & 1 \\
\hline$t_{3}$ & $w_{3}^{1}$ & $r_{4}$ & 2 \\
\hline \multirow{2}{*}{$t_{4}$} & $w_{4}^{1}$ & $r_{1}, r_{3}$ & 4 \\
& $w_{4}^{2}$ & $r_{2}, r_{4}$ & 3 \\
& $w_{4}^{3}$ & $r_{2}, r_{3}$ & 7 \\
\hline
\end{tabular}

schemes. It can help the algorithms to match the TTSP with multiple alternative schemes. 
TABLE 3: Example of the integrated encoding scheme.

\begin{tabular}{lcccc}
\hline Decision variables $x_{i j}$ & 0.8147 & 0.9058 & 0.1270 & 0.6324 \\
\hline Tast sequence $t_{j}$ & 3 & 4 & 1 & 2 \\
$k$ & 1 & 1 & 2 & 1 \\
$w_{j}^{k}$ & $r_{4}$ & $r_{1}, r_{3}$ & $r_{2}, r_{4}$ & $r_{1}$ \\
$P_{j}^{k}$ & 2 & 4 & 3 & 4 \\
\hline
\end{tabular}

TABLE 4: The integrated encoding scheme.

\begin{tabular}{lccc}
\hline & {$[0.0,0.1)$} & {$[0.1,0.2)$} & {$[0.2,0.3)$} \\
Decision & {$[0.3,0.4)$} & {$[0.4,0.5)$} & {$[0.5,0.6)$} \\
variables $x_{i j}$ & {$[0.6,0.7)$} & {$[0.7,0.8)$} & {$[0.8,0.9)$} \\
& {$[0.9,1.0)$} & 2 & 3 \\
\hline$k$
\end{tabular}

5.2. Application of Chaotic Maps in MOEA/D. The multiobjective evolutionary algorithm based on decomposition is originated from Tchebycheff decomposition. It decomposes a multiobjective problem into a number of scalar optimization subproblems and optimizes them simultaneously. Each subproblem is bound with a weight vector and is optimized by using the information from its several neighbor subproblems [20].

In this paper, chaotic variables are used instead of random variables in MOEA/D. Ten chaotic maps are embedded in MOEA/D to replace the random operation. Three key phases in evolutionary algorithms, initialization, crossover, and mutation, are chosen to be embedded with chaos. Different chaotic maps have different formulas and characters. Here, we use sinusoidal map [21] as an example.

(1) Initialization. In order to guarantee the diversity of the initial population, the chaos initialization is applied in this paper.

For example, we assume $N$ individuals in population, and one of them can be denoted by

$x_{s}=\left\{x_{s}^{1}, x_{s}^{2}, \ldots x_{s}^{i}, \ldots, x_{s}^{n}\right\}, \quad s=1,2, \ldots, N, i=1,2, \ldots, N$.

Here, the initial population is generated by chaos maps. For example, if the sinusoidal map is used for initialization, $x_{s}^{i+1}=\sin \left(\pi x_{s}^{i}\right)$.

(2) Crossover. Crossover is the most important step in the process of the evolution. It is directly related to the convergence, diversity, and other performances of the optimal solutions.

In this paper, a differential evolution (DE) operator is adopted. In the DE operator, each child individual $x_{i}^{t+1}$ is generated as follows:

$$
x_{i}^{t+1}= \begin{cases}x_{i}^{t}+F \times\left(x_{i 1}^{t}-x_{i 2}^{t}\right) & \text { if rand }<\mathrm{CR} \\ x_{i}^{t} & \text { otherwise }\end{cases}
$$

Here, $\mathrm{CR}$ and $F$ are two control parameters. $x_{i 1}^{t}$ and $x_{i 2}^{t}$ are two individuals chosen in the neighborhood of $x_{i}^{t}$. Since $F$ is a random number that ranges from 0 to $1, F$ can be generated by chaotic maps instead of random generation. For instance, if the sinusoidal map is used and, in the $i$ th iteration, $F=F_{i}$, then, in the $(i+1)$ th iteration, $F_{s}=F_{i+1}=\sin \left(\pi F_{i}\right)$.

(3) Mutation. Mutation operator that prevents solutions from being trapped into local optima is indispensable in the process of the evolution.

In this paper, a polynomial mutation operator is adopted. For a solution $x_{s}$, the polynomial mutation is described as

$$
x_{s}^{*}=x_{s}+\left(x_{s}^{u}-x_{s}^{l}\right) \times \delta_{s}
$$

where $x_{s}^{u}$ and $x_{s}^{l}$ are the upper and lower bounds of $x_{s}$. Consider

$$
\delta_{s}= \begin{cases}\left(2 u_{s}\right)^{1 /\left(\eta_{m}+1\right)}-1 & \text { if } u_{s}<0.5 \\ 1-\left(2 \times\left(1-u_{s}\right)\right)^{1 /\left(\eta_{m}+1\right)} & \text { otherwise. }\end{cases}
$$

Here, $u_{s}$ is a random number ranging from 0 to $1 . \eta_{m}$ is the distribution index for the mutation operator. Similar to the crossover scheme, we have $u_{s}=u_{i+1}=\sin \left(\pi u_{i}\right)$ when using the sinusoidal map.

\section{Experiments}

We carry out four types of experiments to illustrate the performances of the mentioned approaches. Experiment 1 shows the effectiveness of the improving encoding method based on one large scale TTSP. Experiment 2 aims to solve a small scale TTSP benchmark to measure the performance of the evolutionary algorithm using chaotic maps in three phases. Experiment 3 is similar to experiment 2, except that it aims to solve the large scale TTSP. In both experiments 2 and 3 , ten chaotic maps are embedded in three different phases in the original MOEA/D algorithm. Each time, only one parameter is modified. The Pareto set (PF) is used to show the effect firstly. Then, the performance metrics HV and $C$ are used to further evaluate the performance of chaotic maps embedded algorithm and the original algorithm. Based on the results of the above experiments, we compare the CMOEA/D with the VNM [5] in experiment 4.

The parameters for all experiments are shown in Table 6. $n_{\text {iter }}$ is the number of iterations. $n_{\text {pop }}$ is the scale of the population. $n_{\mathrm{var}}$ is the number of decision variables. CR and $P_{m}$ (equal to the reciprocal of $n_{\mathrm{var}}$ ) are the probabilities of crossover and mutation operations.

6.1. Experiment 1: The Performance of the Improving Encoding Method. This experiment shows the effectiveness of the improving encoding method in solving the TTSP. The instance is based on a large scale TTSP $40 \times 12$ [4]. 50 runs of the same experiment have been performed, and the best run among the 50 runs is given in Figure 2. Here, MOEA/D1, MOEA/D-2, and MOEA/D-3 represent the algorithm with different encoding method of random, IES, improving IES separately.

We can find from the Pareto front that the improving encoding method obtains better convergence of the solutions of the TTSP. The equal probability also helps the algorithm 
TABLE 5: The improving integrated encoding scheme.

\begin{tabular}{lccc}
\hline & {$[0,1)[3,4)[6,7)[9,10)$} & {$[1,2)[4,5)[7,8)[10,11)$} & {$[2,3)[5,6)[8,9)[11,12)$} \\
Decision variables $x_{i j} / 30$ & {$[12,13)[15,16)[18,19)$} & {$[13,14)[16,17)[19,20)$} & {$[14,15)[17,18)[20,21)$} \\
& {$[21,22)[24,25)[27,28)$} & {$[22,23)[25,26)[28,29)$} & {$[23,24)[26,27)[29,30)$} \\
\hline$k$ & 1 & 2 & 3 \\
\hline
\end{tabular}

TABLE 6: The setting of parameters.

\begin{tabular}{|c|c|c|c|c|}
\hline & $6 \times 8$ & $20 \times 8$ & $30 \times 12$ & $40 \times 12$ \\
\hline$n_{\text {iter }}$ & & & & \\
\hline$n_{\text {pop }}$ & & & & \\
\hline$n_{\mathrm{var}}$ & 6 & 20 & 30 & 40 \\
\hline CR & & & & \\
\hline$P_{m}$ & $1 / 6$ & $1 / 20$ & $1 / 30$ & $1 / 40$ \\
\hline
\end{tabular}

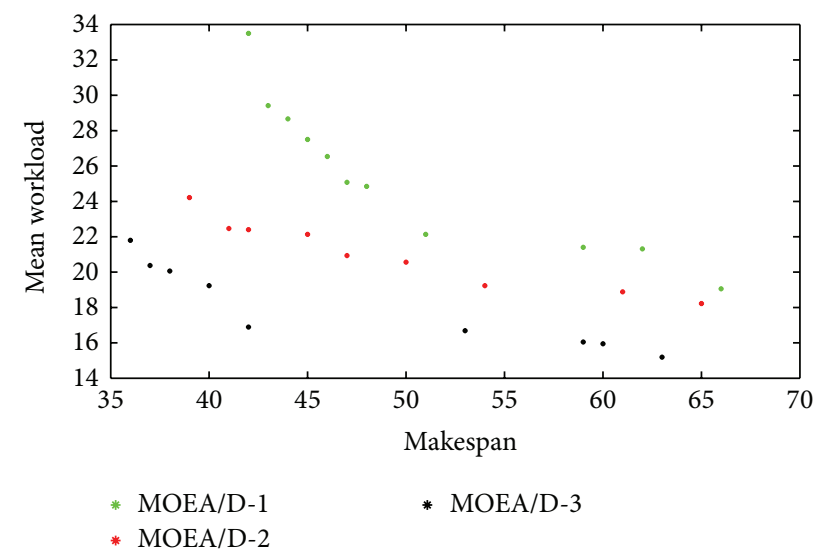

Figure 2: Comparison of different encoding methods in solving the TTSP.

to obtain good convergence. Therefore, the improving IES is used in the following experiments because of the efficiency.

6.2. Experiment 2: The Performance for the Small Scale TTSP. This experiment is carried out to show the effectiveness of CMOEA/D for the small scale TTSP $6 \times 8.10$ times of the same experiment have been performed, and the best results obtained from original MOEA/D and many variants of CMOEA/D for this instance are shown in Figures 3, 4, and 5.

For the convenience, the algorithms with different combinations of chaotic maps and phases are named as "CMOEA/D-[phase][chaotic map]." The ten chaotic maps (baker, cat, circle, cubic, Gauss, ICMIC, logistic, sinusoidal, tent, and Zaslavskii) are denoted by $1,2,3, \ldots, 10$ in alphabetical order. " $R$ " represents the original MOEA/D. " $I$ " represents the phase for initial population. " $C$ " represents the phase for the crossover operator. " $M$ " represents the phase for the mutation operator. For example, the algorithm for initial population by logistic map is named "CMOEA/D-I7"

According to the name role, Figure 3 indicates the performance of the chaotic maps for crossover for solving the TTSP. Figure 4 shows the performance of the chaotic maps for initialization for solving the TTSP. Figure 5 shows the performance of the chaotic maps for mutation for solving the TTSP.

For the small scale TTSP, the performance for convergence is not very obvious from the Pareto set. The solutions obtained from the original algorithm and the chaos embedded algorithm overlap each other. However, the diversity of the solutions obtained from the chaos embedded algorithm is better than the original algorithm.

6.3. Experiment 3: The Performance for the Large Scale TTSP. This experiment is carried out to show the effectiveness of CMOEA/D for three large scale problems, TTSP $20 \times 8$, $30 \times 12$, and $40 \times 12.10$ times of the same experiment have been performed, and the best results obtained from original MOEA/D and many variants of CMOEA/D are shown in Figures $6,7,8,9,10,11,12,13$, and 14 . The name role of the figures is similar to the small scale instance.

For the large scale TTSP, both the convergence and diversity of solutions are improved significantly. Almost every chaotic map has good performance for the improvement, but the performance is not stable and positive for some chaotic maps. For example, the tent, baker, and cat maps even have negative effects for the solutions under some situations.

6.4. Performance Analysis. Based on the above experiments, we use the statistical data of the comprehensive metric $\mathrm{HV}$ and convergence metric $C$ to indicate the results from different aspects, because the figure of Pareto front can provide only the primary idea but not the comprehensive effect. The conclusion about the guidance of chaotic maps for resolving the TTSP will be investigated based on these data.

\subsubsection{Performance Metrics}

(1) Hypervolume (see [11]). This quality indicator calculates the volume (in the objective space) covered by members of a nondominated set of solutions for problems where all objectives are to be minimized. Mathematically, for each solution $i \in S$, a hypercube $v_{i}$ is constructed with a reference point $W$ and the solution $i$ as the diagonal corners of the hypercube. The reference point can simply be found by constructing a vector of worst objective function values. Thereafter, a union of all hypercubes is found and its hypervolume is calculated as follows:

$$
\operatorname{HV}(S)=\operatorname{Leb}\left(\bigcup_{i \in S} v_{i}\right)
$$



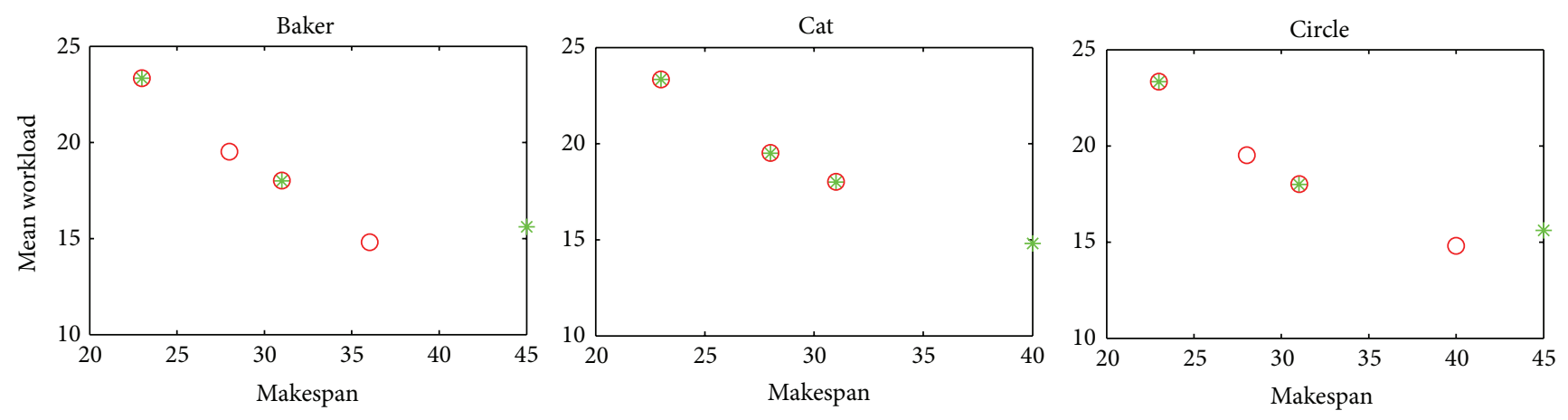

* MOEA/D

* MOEA/D

* MOEA/D

O CMOEA/D-C1

O CMOEA/D-C2
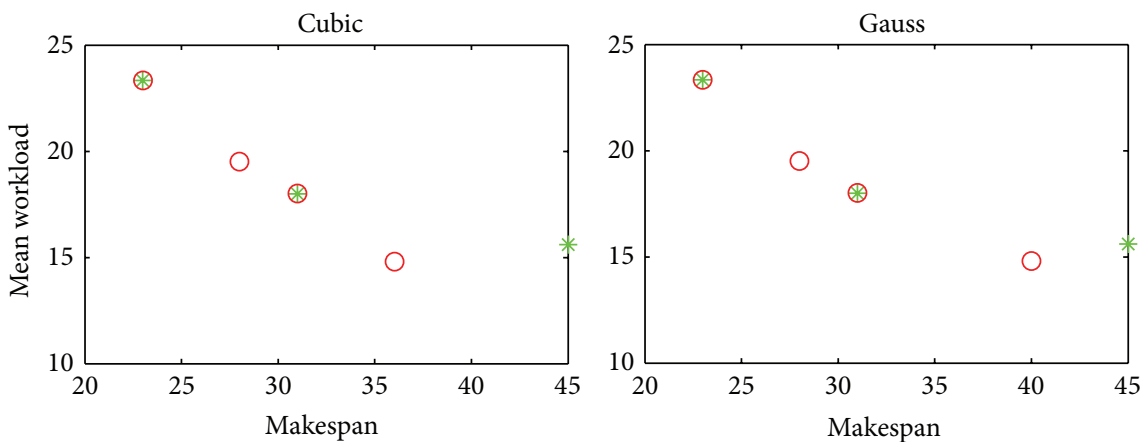

O CMOEA/D-C3

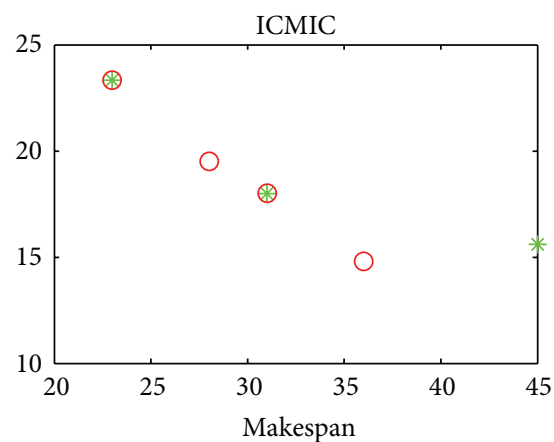

* MOEA/D

O CMOEA/D-C4

* MOEA/D

O CMOEA/D-C5

* MOEA/D

O CMOEA/D-C6
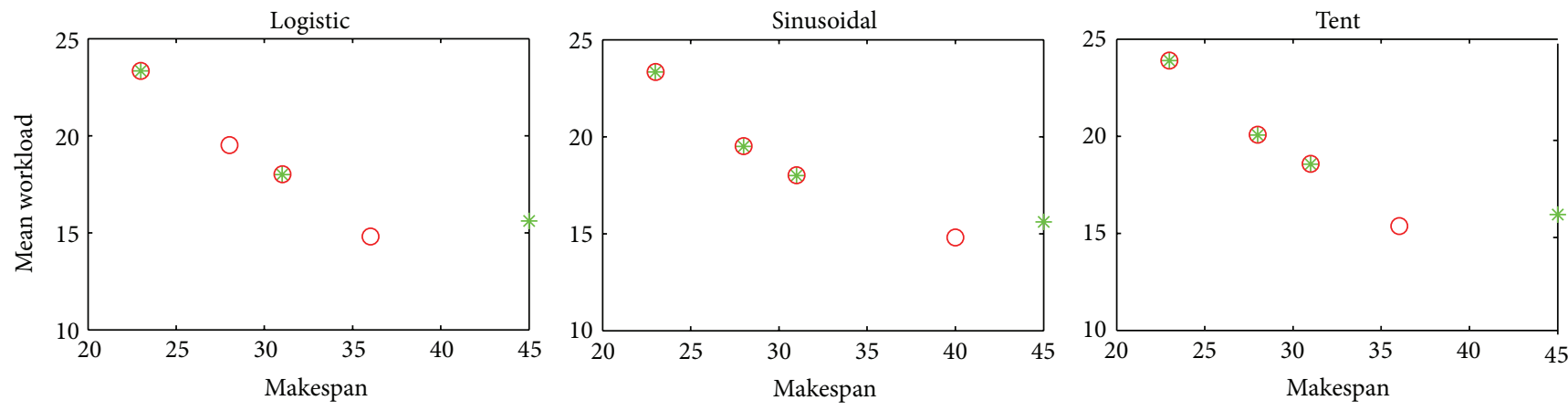

* MOEA/D

* MOEA/D

O CMOEA/D-C8

* MOEA/D

O CMOEA/D-C7

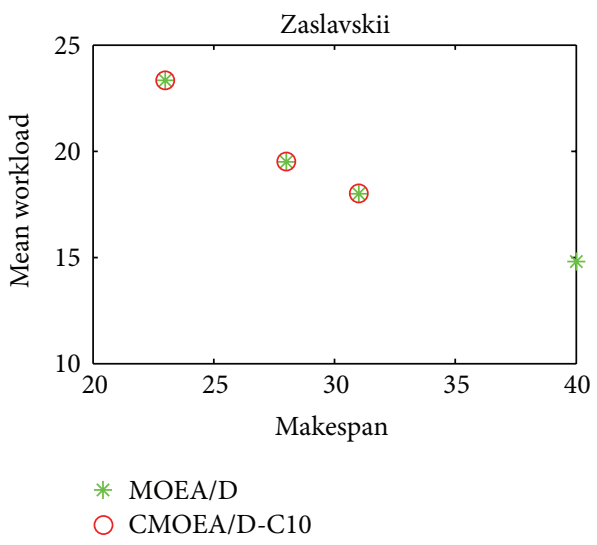

FIgURE 3: Comparison of different chaotic maps for crossover. 

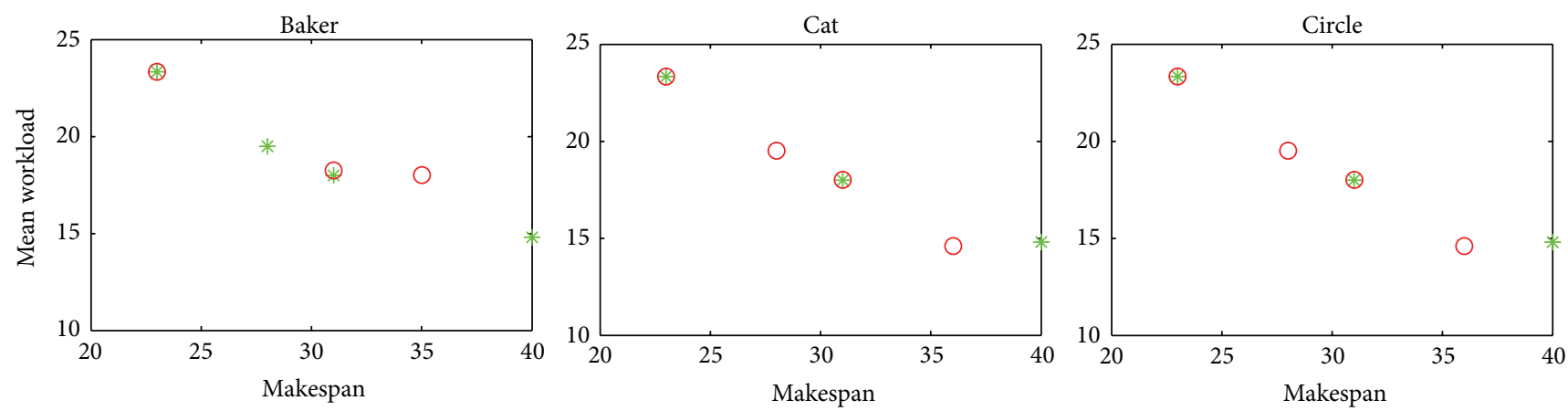

* MOEA/D

O CMOEA/D-I1

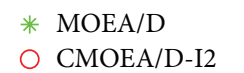

* MOEA/D

O CMOEA/D-I3
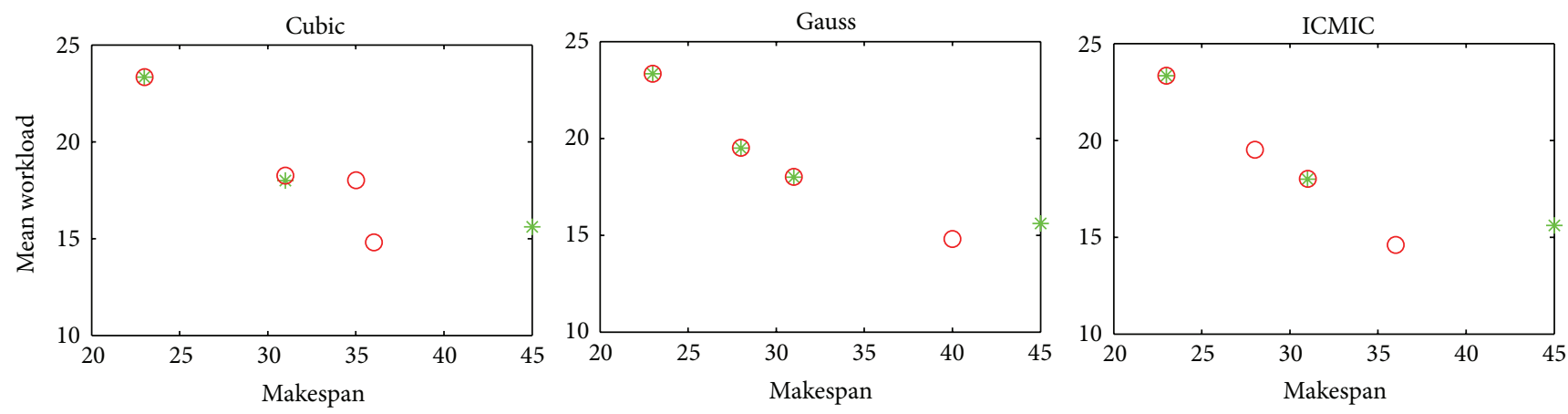

* MOEA/D

* MOEA/D

O CMOEA/D-I4

O CMOEA/D-I5

* MOEA/D

O CMOEA/D-I6
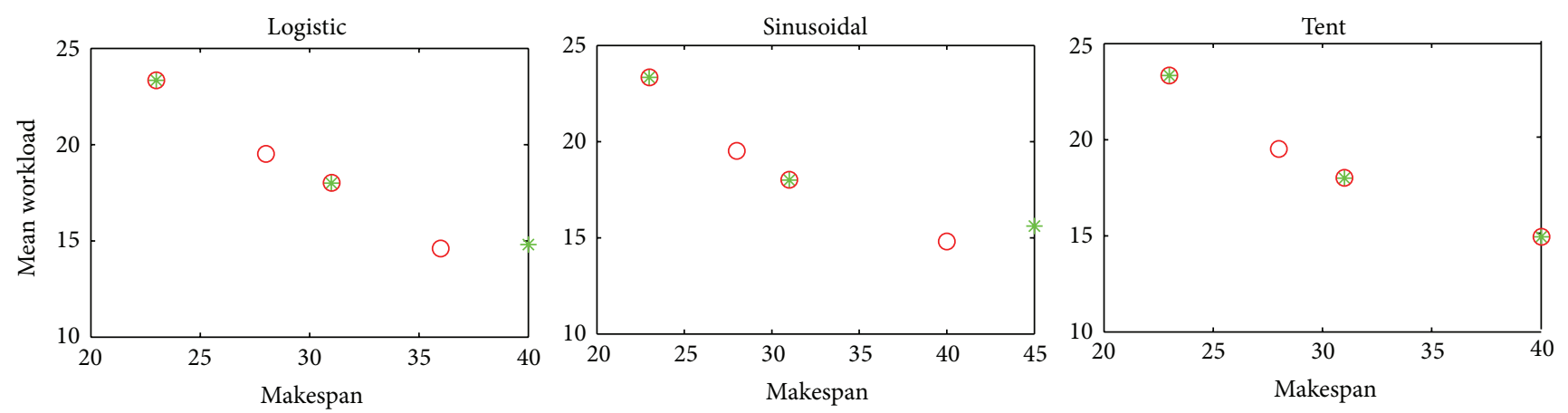

* MOEA/D

* MOEA/D

* MOEA/D

O CMOEA/D-I7

O CMOEA/D-I8

O CMOEA/D-I9

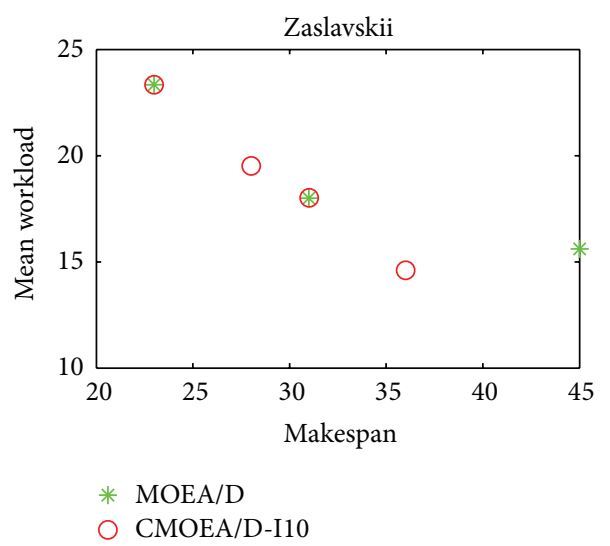

FIgURE 4: Comparison of different chaotic maps for initialization. 

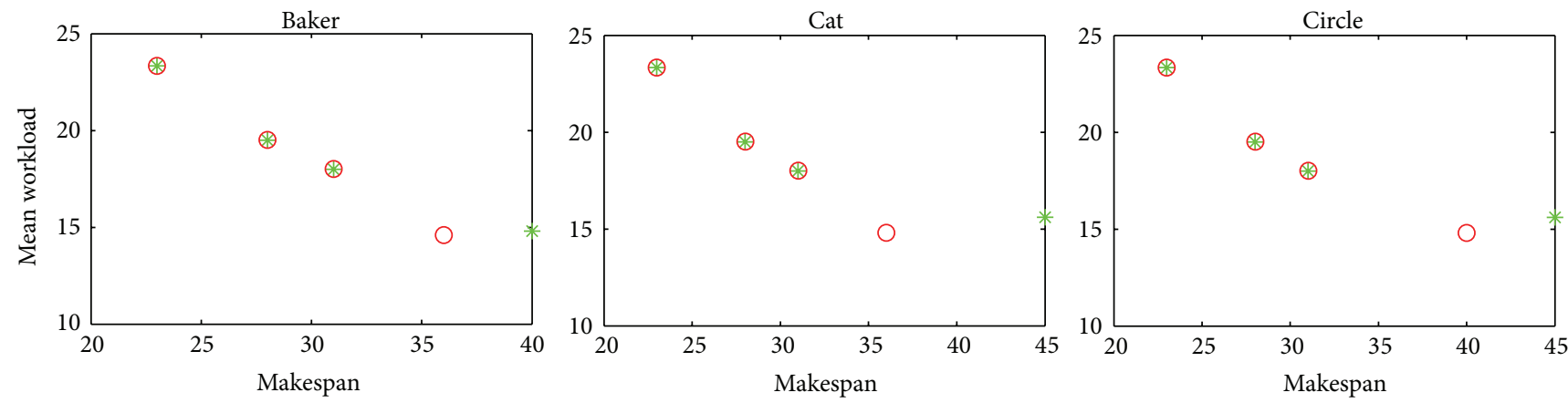

* MOEA/D

O CMOEA/D-M1

* MOEA/D

* MOEA/D

O CMOEA/D-M2

O CMOEA/D-M3
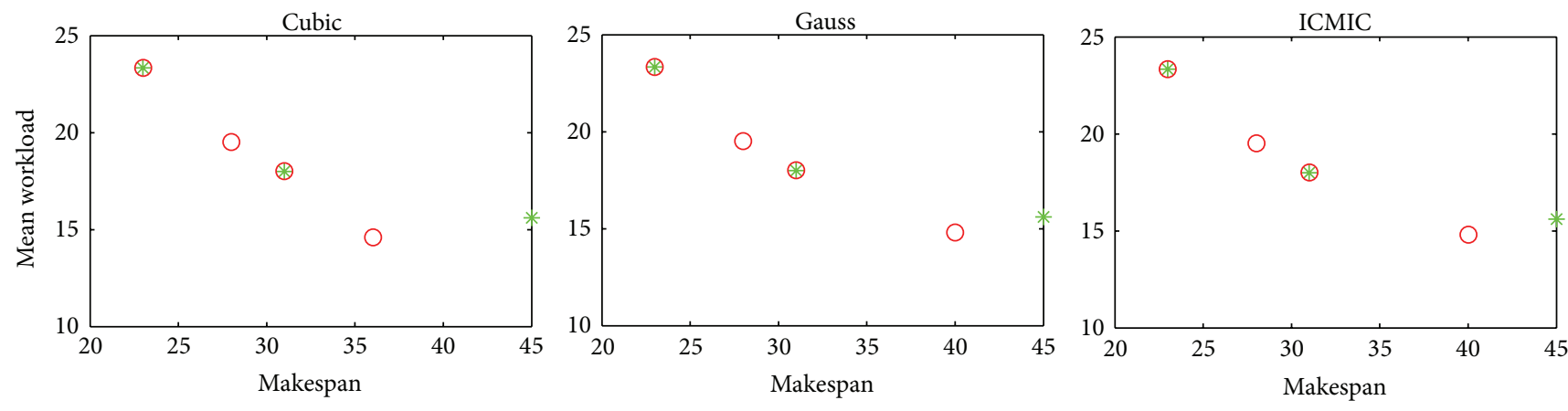

* MOEA/D

* MOEA/D

* MOEA/D

O CMOEA/D-M4

O CMOEA/D-M5

O CMOEA/D-M6
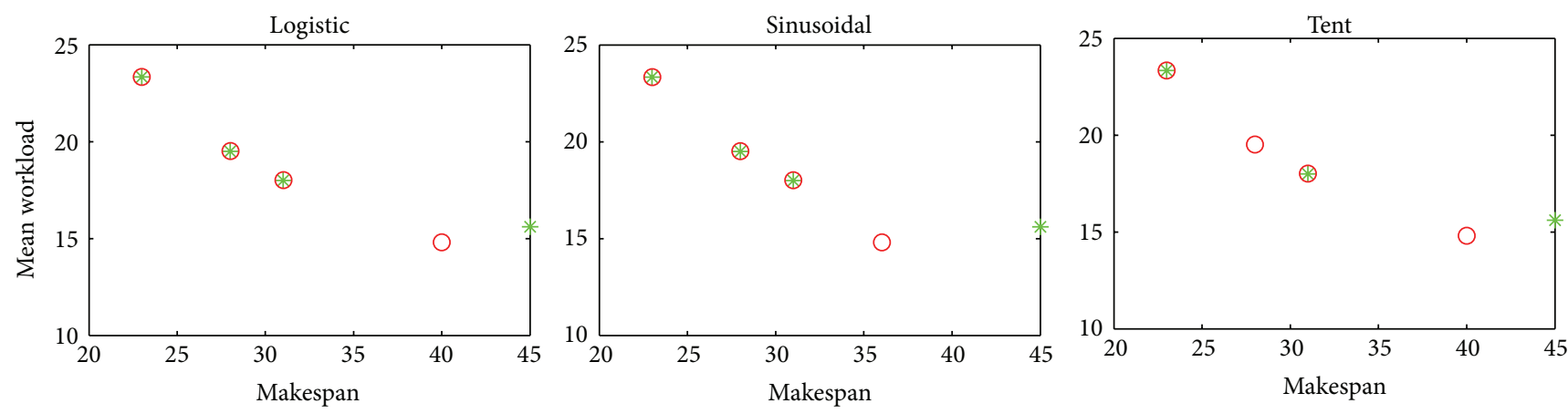

* MOEA/D

* MOEA/D

* MOEA/D

O CMOEA/D-M7

O CMOEA/D-M8

O CMOEA/D-M9

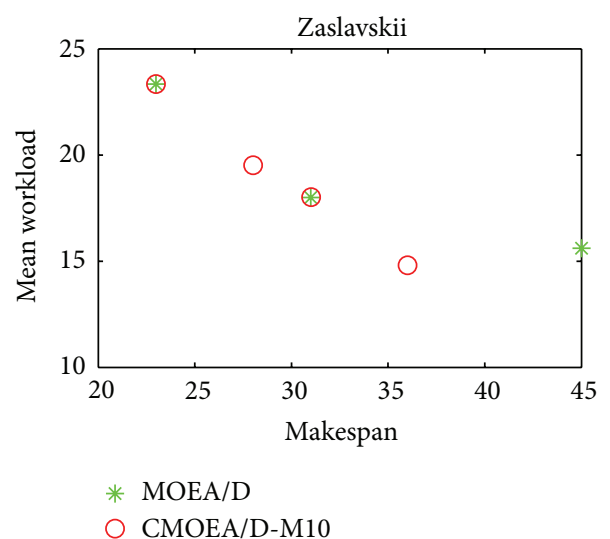

FIgURE 5: Comparison of different chaotic maps for mutation. 

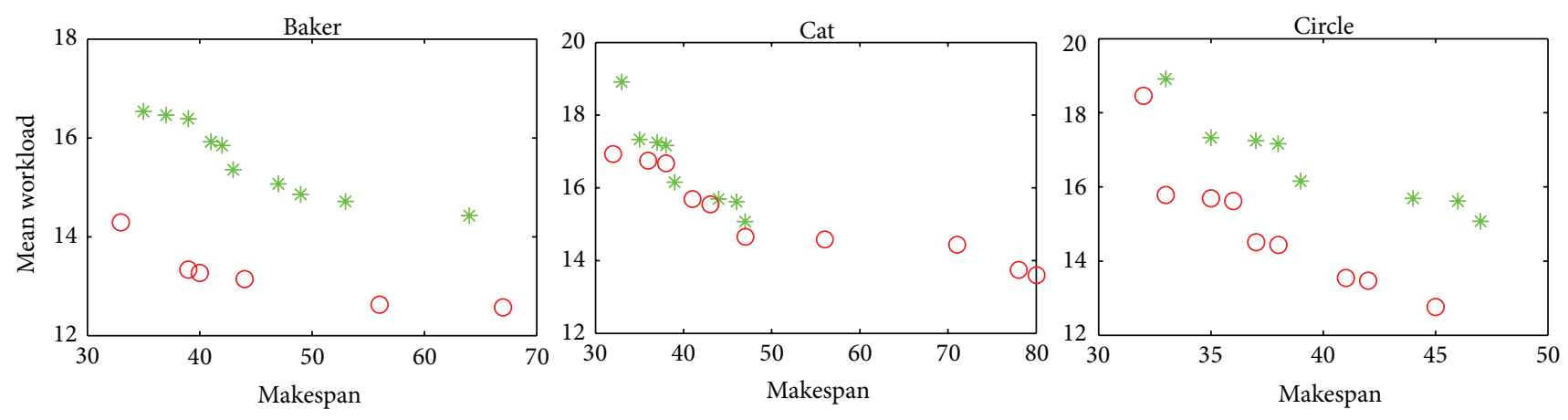

* MOEA/D

* MOEA/D

O CMOEA/D-C1

○ CMOEA/D-C2

* MOEA/D

O CMOEA/D-C3
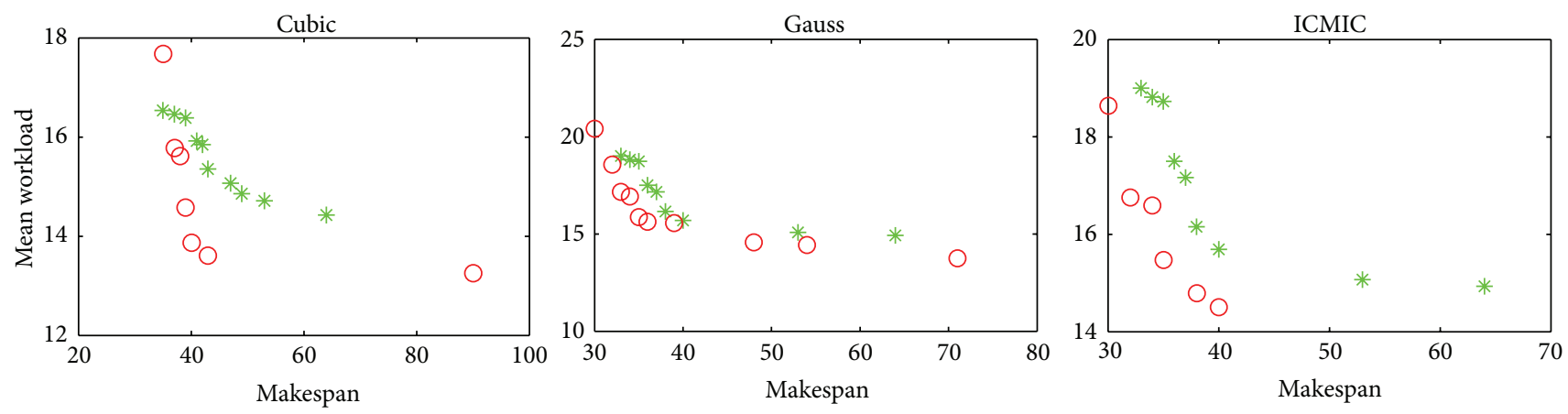

* MOEA/D

* MOEA/D

O CMOEA/D-C4

○ CMOEA/D-C5

* MOEA/D

O CMOEA/D-C6
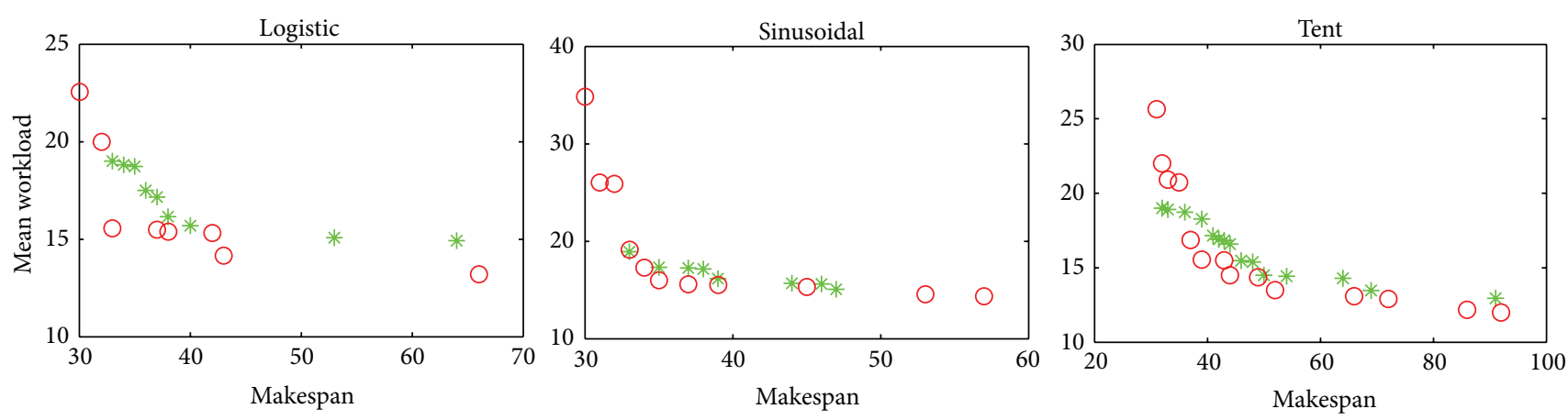

* MOEA/D

* MOEA/D

○ CMOEA/D-C8

* MOEA/D

O CMOEA/D-C7

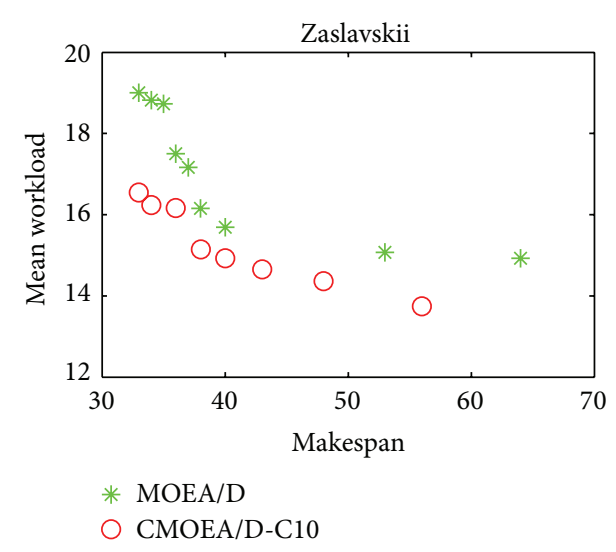

O CMOEA/D-C9

FIgURE 6: Comparison of different chaotic maps for crossover for $20 \times 8$. 

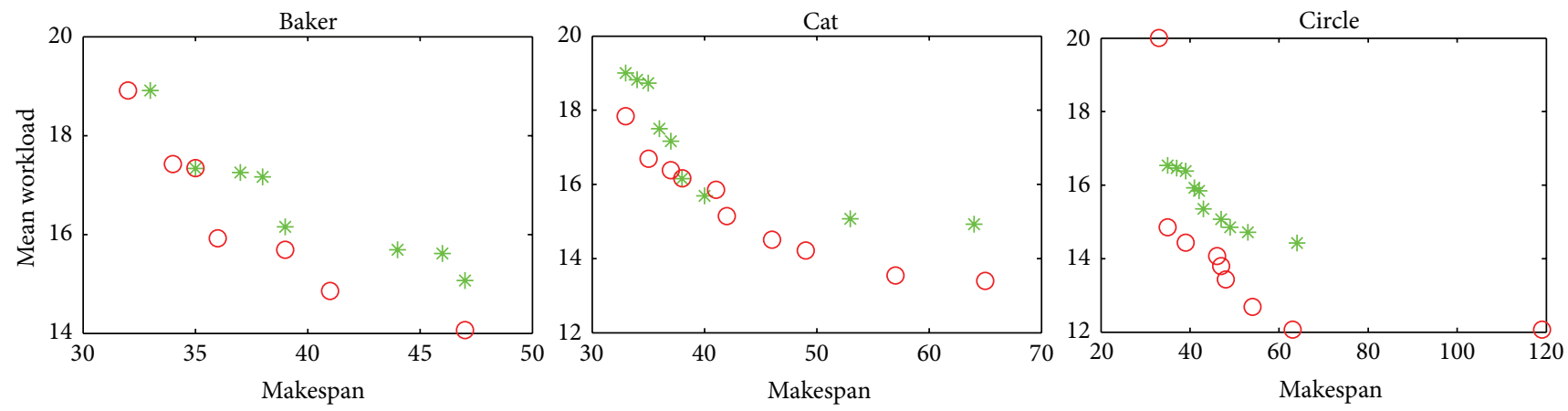

* MOEA/D

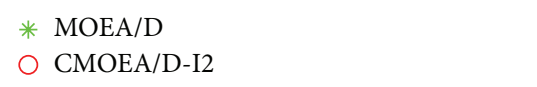

* MOEA/D

O CMOEA/D-I1

O CMOEA/D-I2

O CMOEA/D-I3
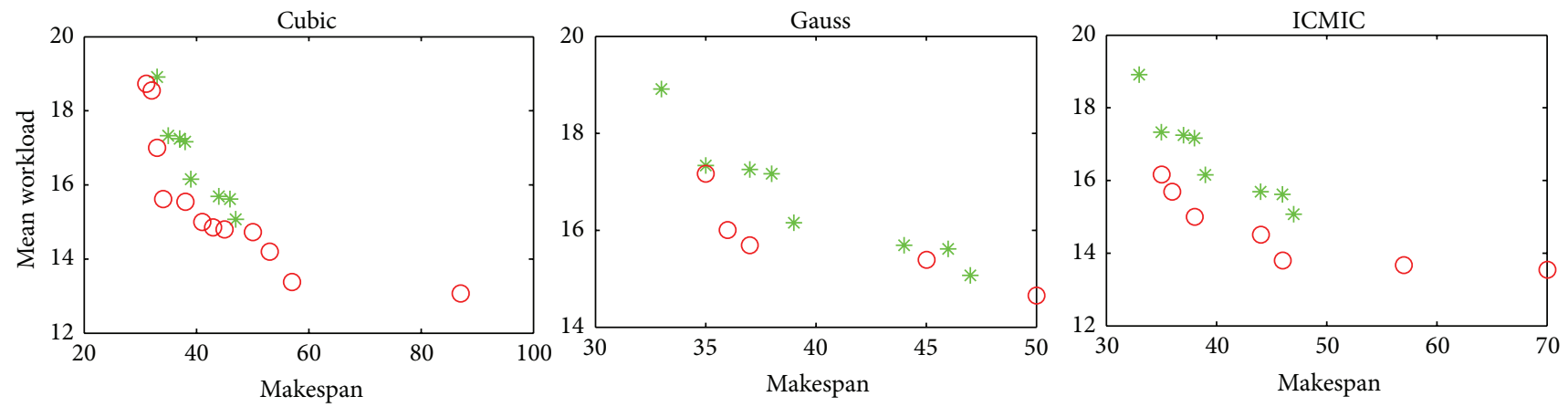

* MOEA/D

O CMOEA/D-I4

* MOEA/D

* MOEA/D

O CMOEA/D-I5

O CMOEA/D-I6
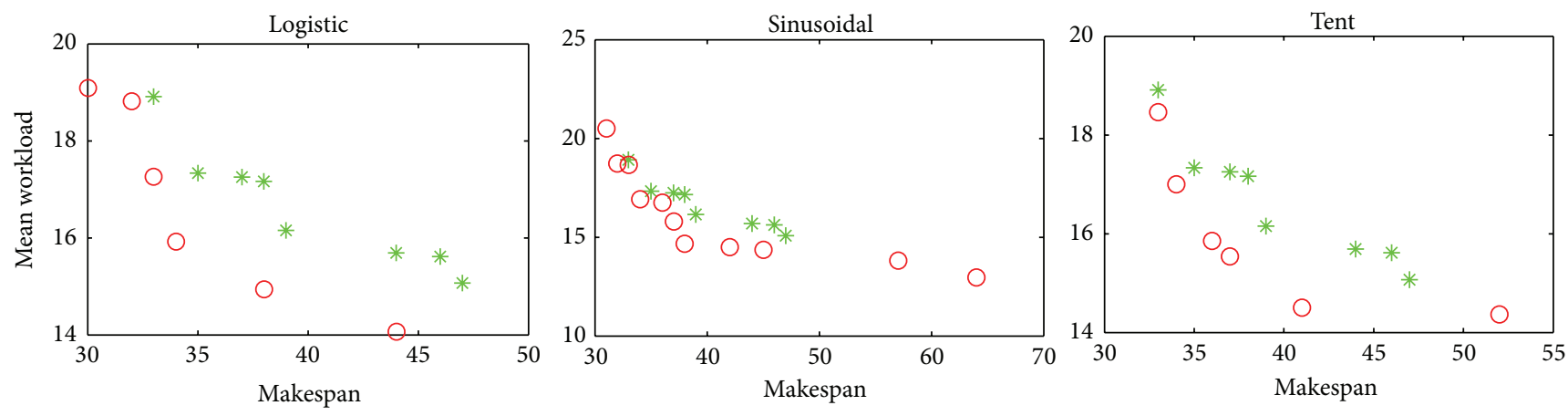

* MOEA/D

* MOEA/D

* MOEA/D

O CMOEA/D-I7

O CMOEA/D-I8

O CMOEA/D-I9

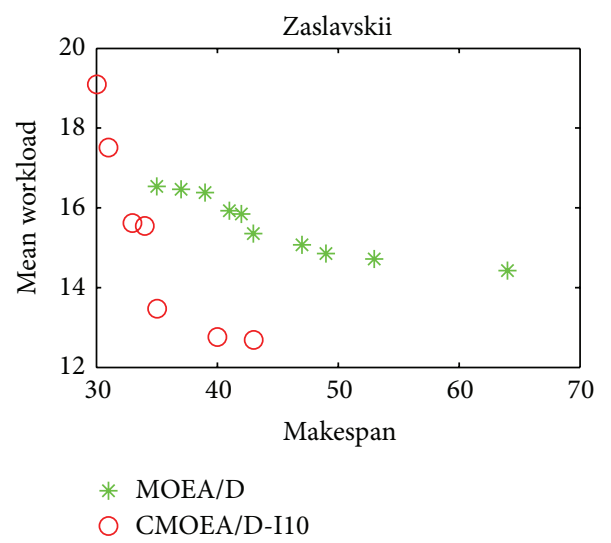

FIGURE 7: Comparison of different chaotic maps for initialization for $20 \times 8$. 

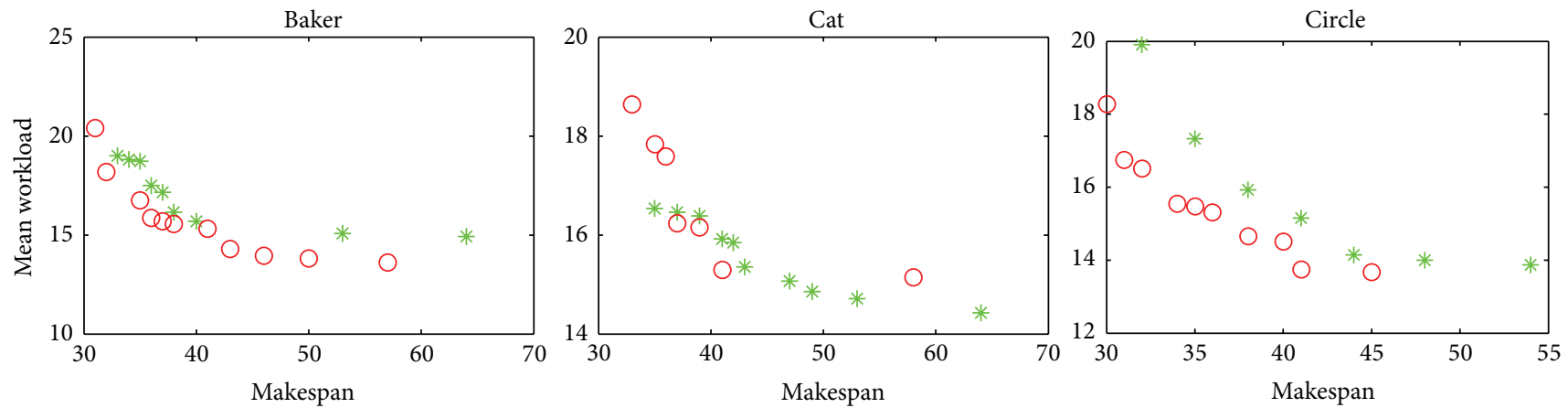

* MOEA/D

O CMOEA/D-M1

* MOEA/D

O CMOEA/D-M2

* MOEA/D

O CMOEA/D-M3
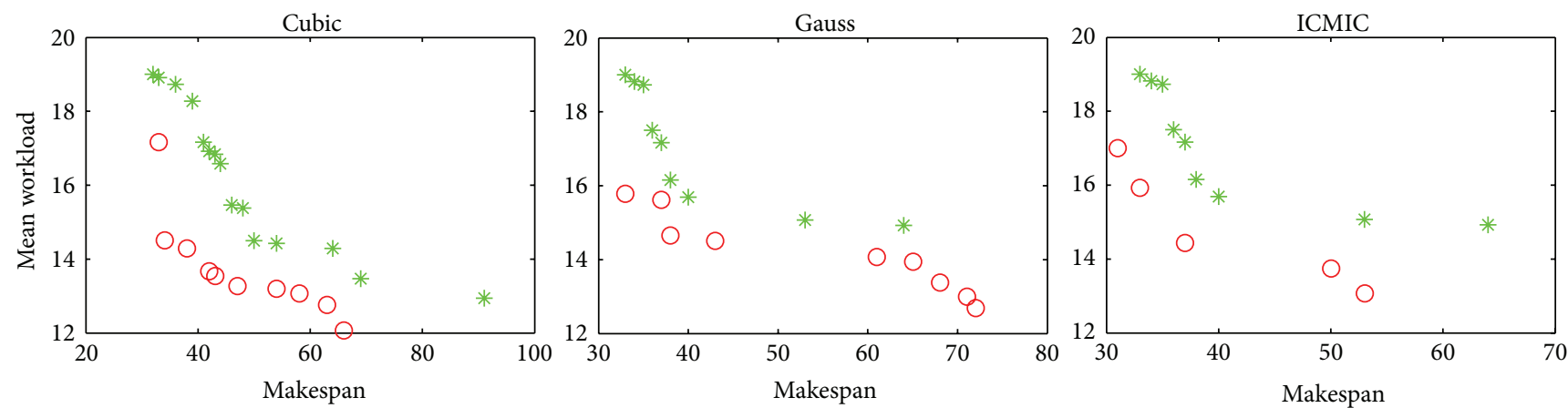

* MOEA/D

* MOEA/D

O CMOEA/D-M5

* MOEA/D

O CMOEA/D-M4

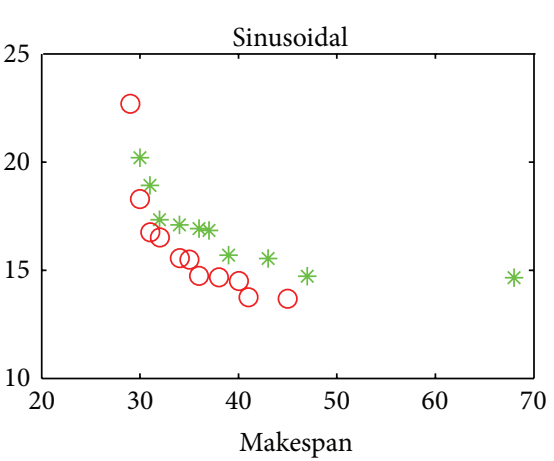

O CMOEA/D-M6

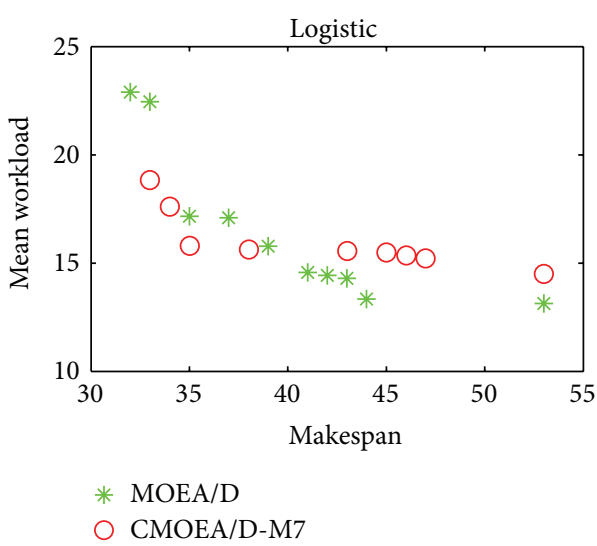

* MOEA/D

O CMOEA/D-M8

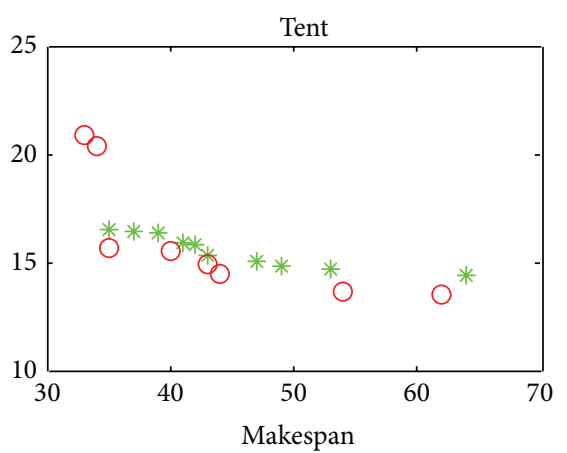

* MOEA/D

O CMOEA/D-M9

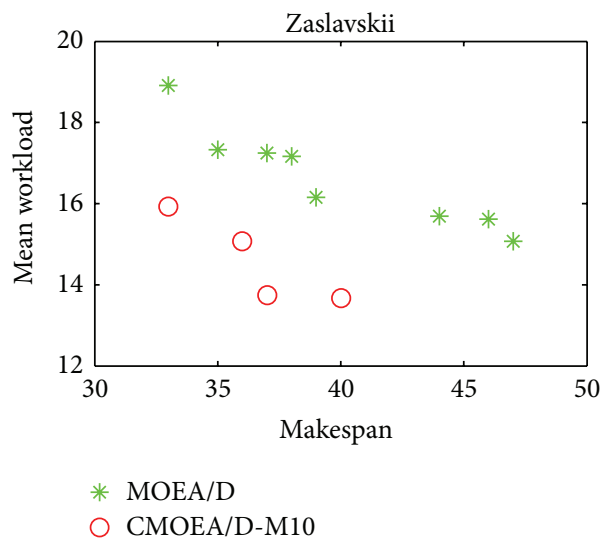

FIGURE 8: Comparison of different chaotic maps for mutation for $20 \times 8$. 

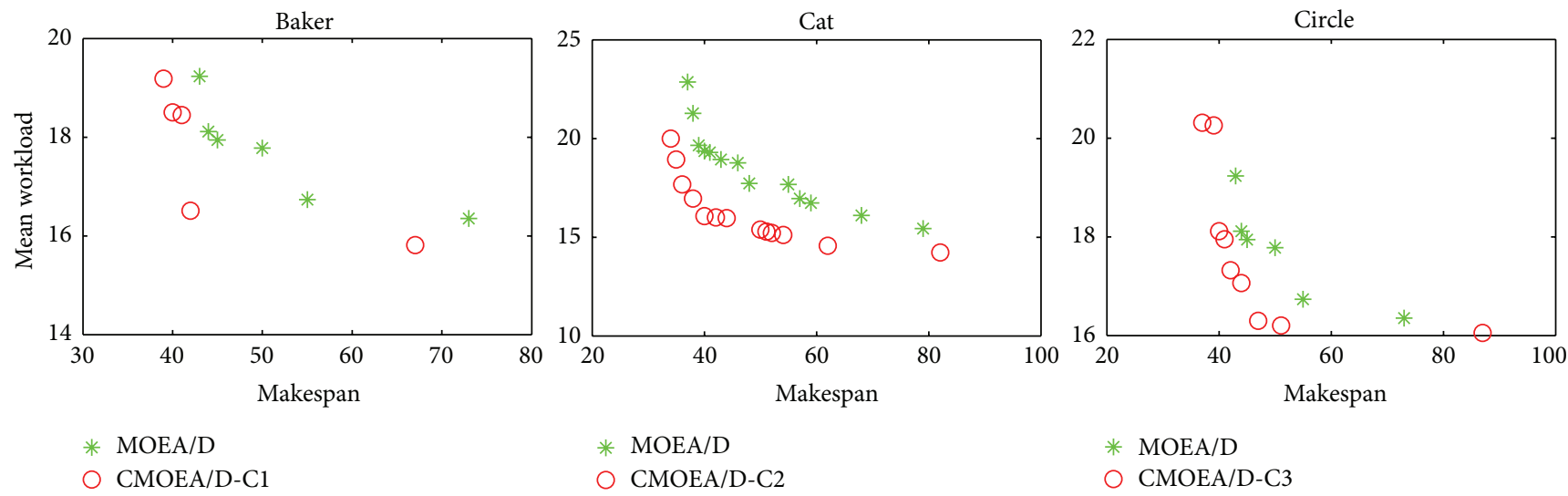

* MOEA/D

O CMOEA/D-C2

* MOEA/D

O CMOEA/D-C3
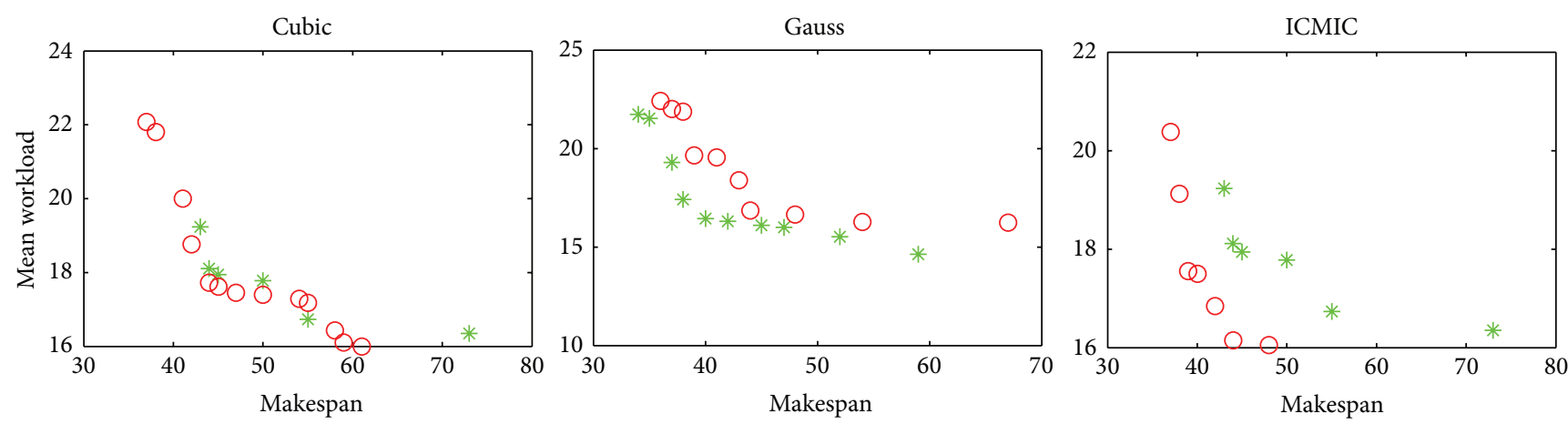

* MOEA/D

* MOEA/D

* MOEA/D

O CMOEA/D-C4

○ CMOEA/D-C5
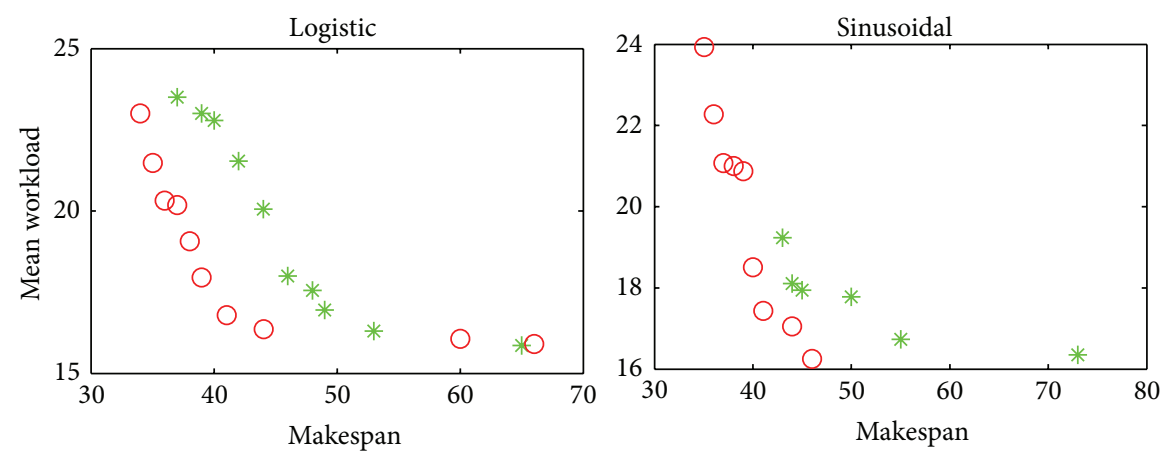

O CMOEA/D-C6

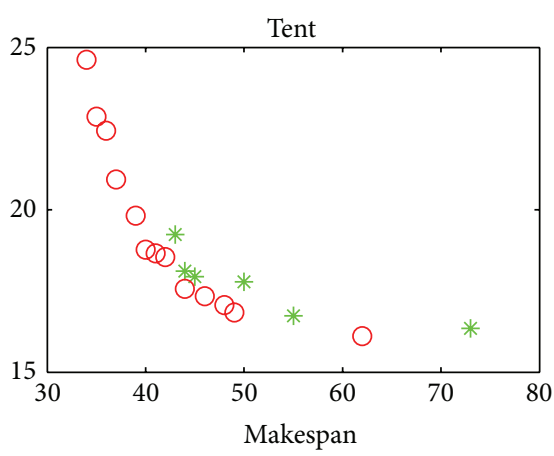
* MOEA/D

* MOEA/D

CMOEA/D-C8

* MOEA/D

O CMOEA/D-C9

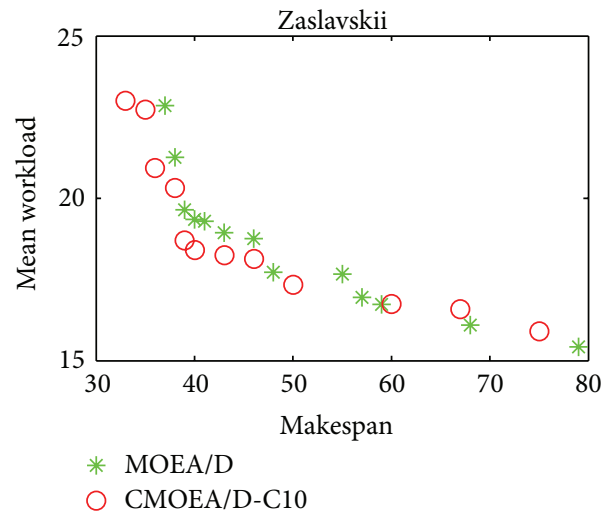

FIGURE 9: Comparison of different chaotic maps for crossover for $30 \times 12$. 

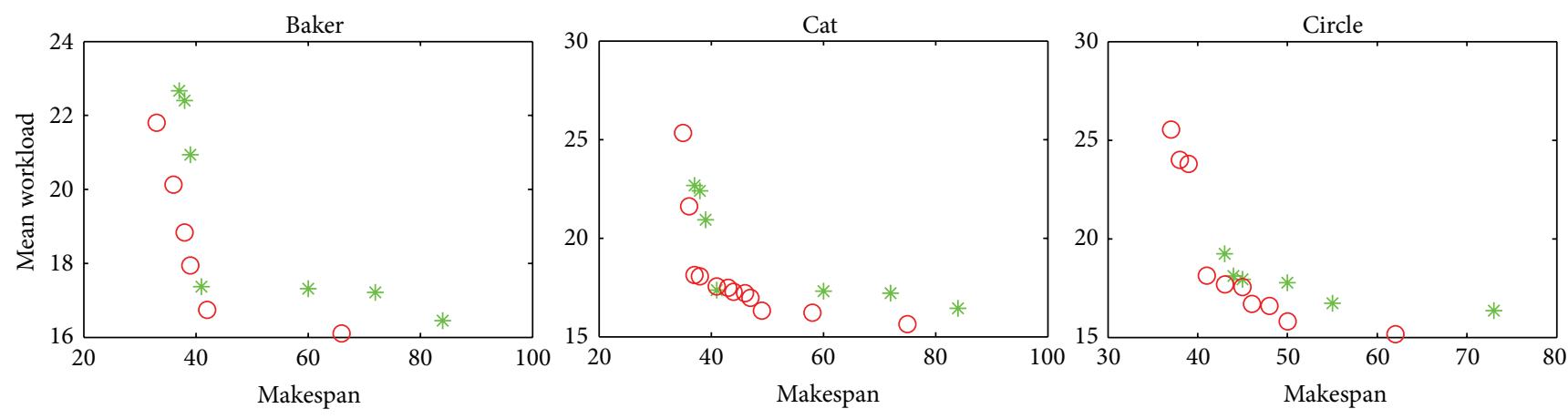

* MOEA/D

* MOEA/D

* MOEA/D

O CMOEA/D-I1

O CMOEA/D-I2

O CMOEA/D-I3
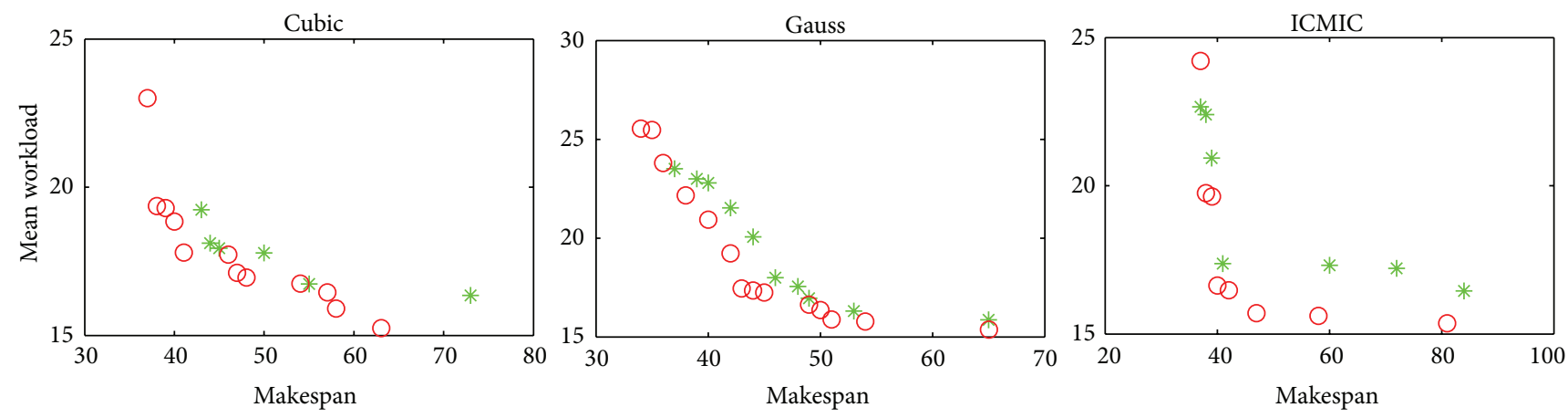

* MOEA/D

○ CMOEA/D-I4

* MOEA/D

O CMOEA/D-I5

* MOEA/D

O CMOEA/D-I6
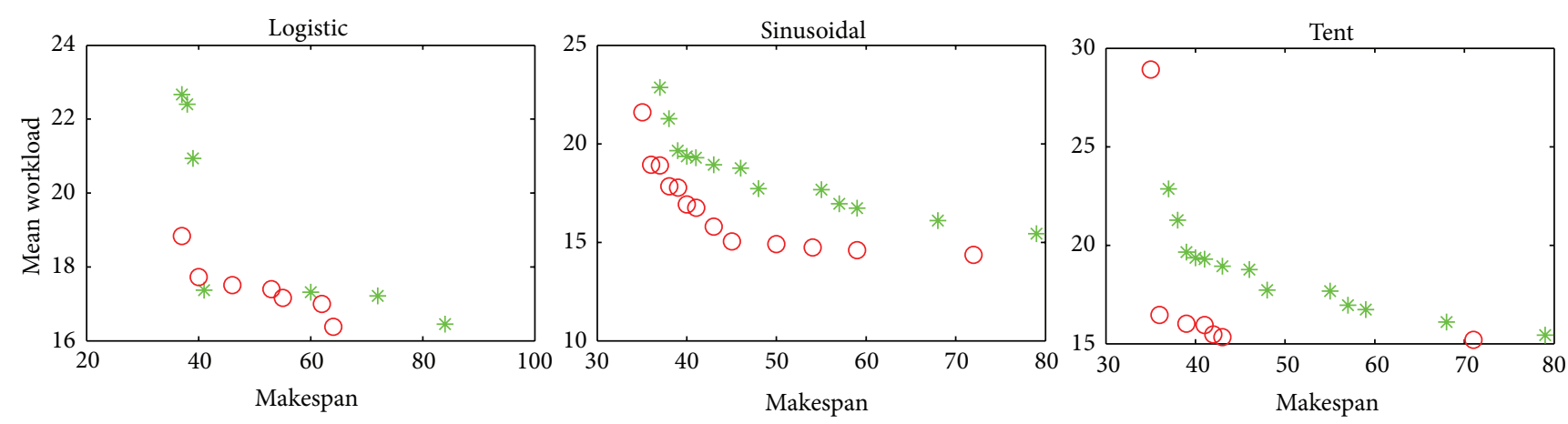
* MOEA/D
O CMOEA/D-I7

* MOEA/D

* MOEA/D

O CMOEA/D-I8

O CMOEA/D-I9

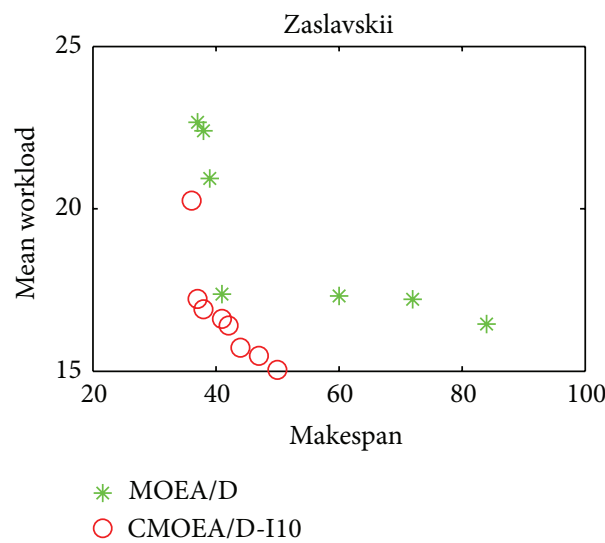

FIgURe 10: Comparison of different chaotic maps for initialization for $30 \times 12$. 

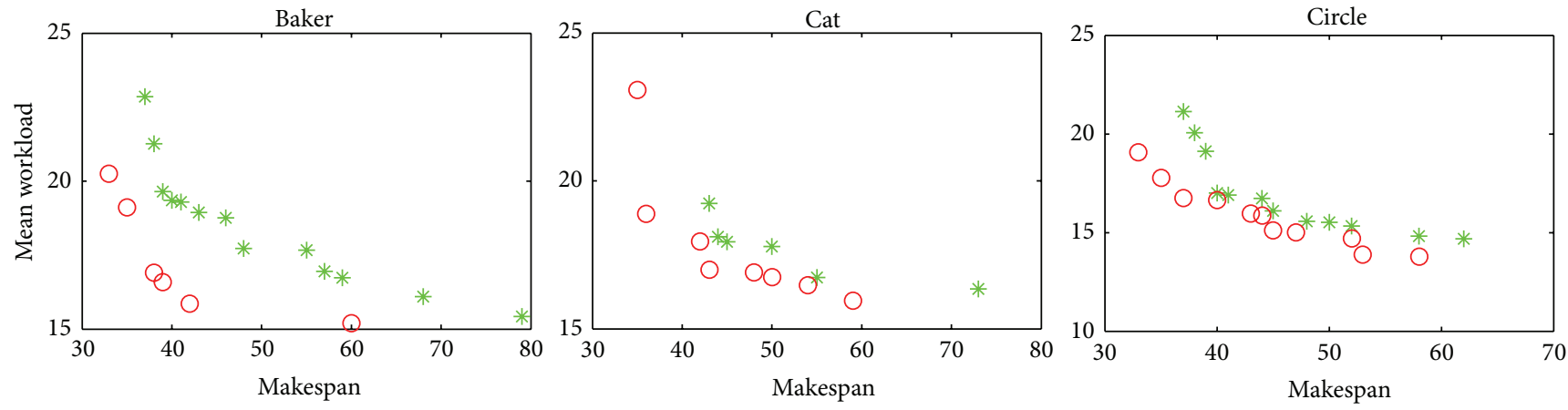

* MOEA/D

O CMOEA/D-M1

* MOEA/D

O CMOEA/D-M2

* MOEA/D

O CMOEA/D-M3
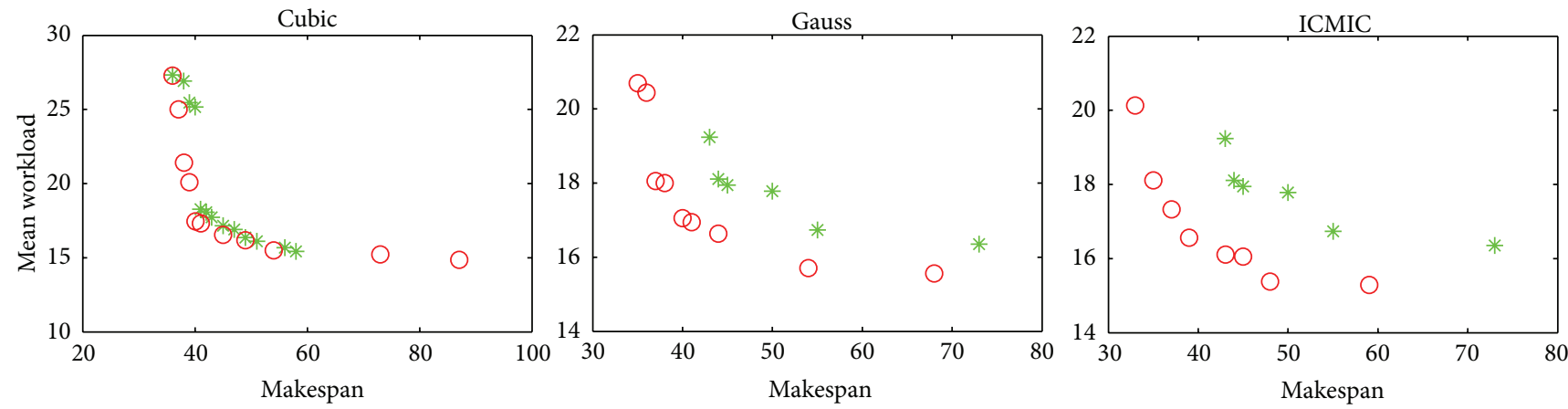

* MOEA/D

O CMOEA/D-M4

* MOEA/D

O CMOEA/D-M5

* MOEA/D

O CMOEA/D-M6
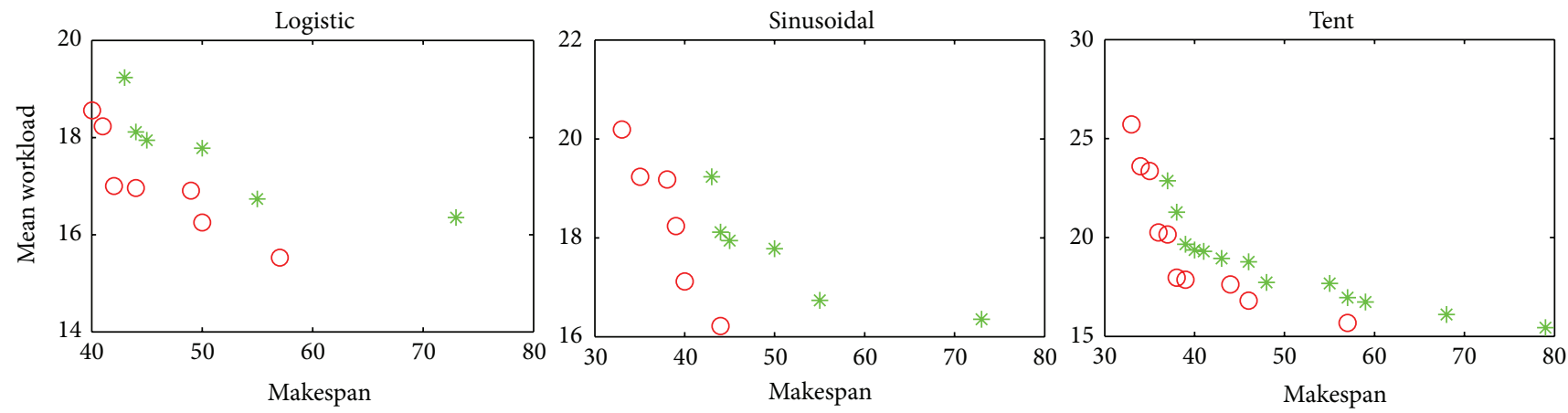

* MOEA/D

* MOEA/D

O CMOEA/D-M8

* MOEA/D

O CMOEA/D-M7

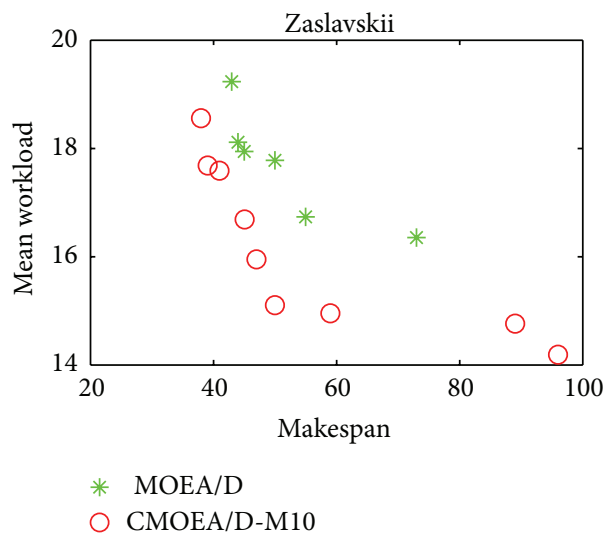

FIGURE 11: Comparison of different chaotic maps for mutation for $30 \times 12$. 

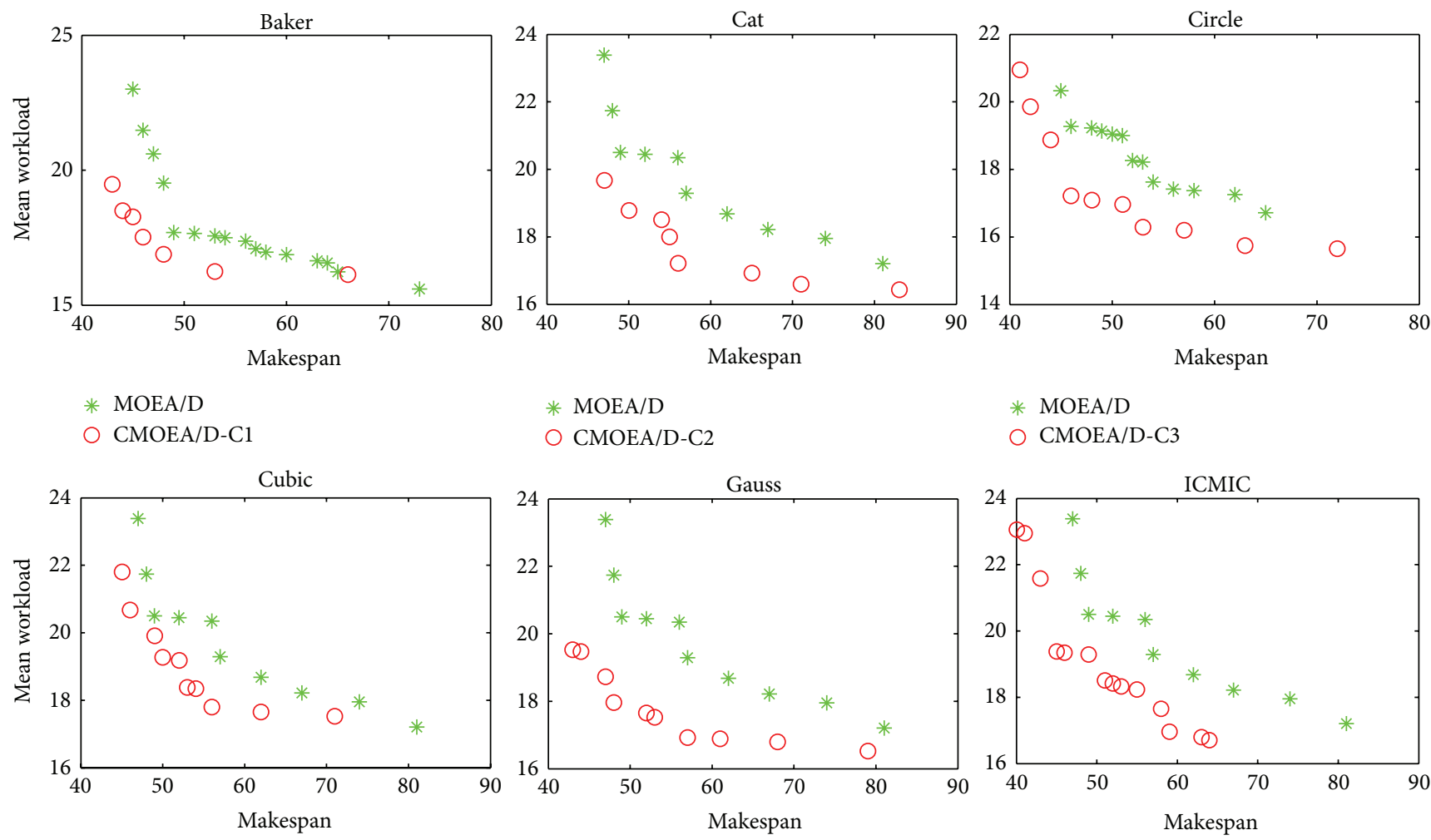

* MOEA/D

O CMOEA/D-C2

O CMOEA/D-C3
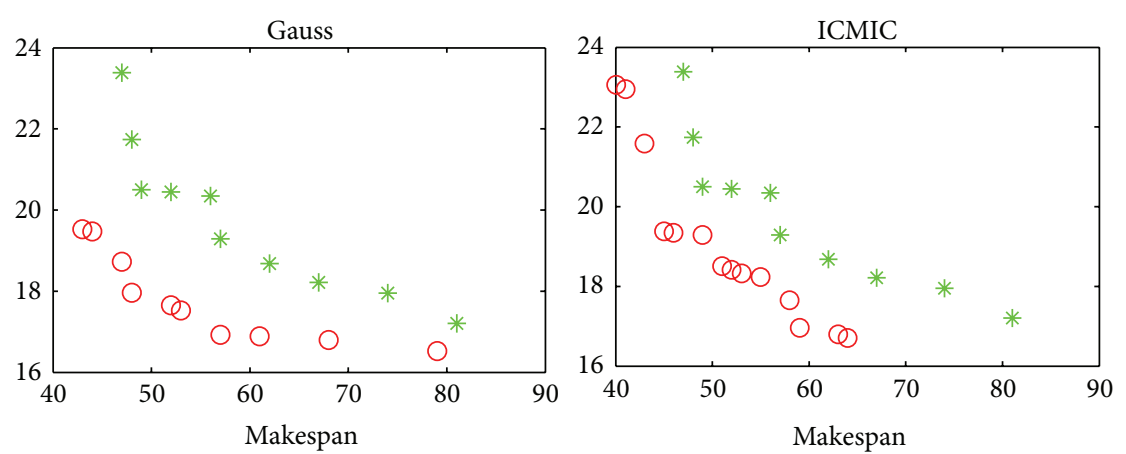

* MOEA/D

O CMOEA/D-C4

* MOEA/D

O CMOEA/D-C5

* MOEA/D

O CMOEA/D-C6
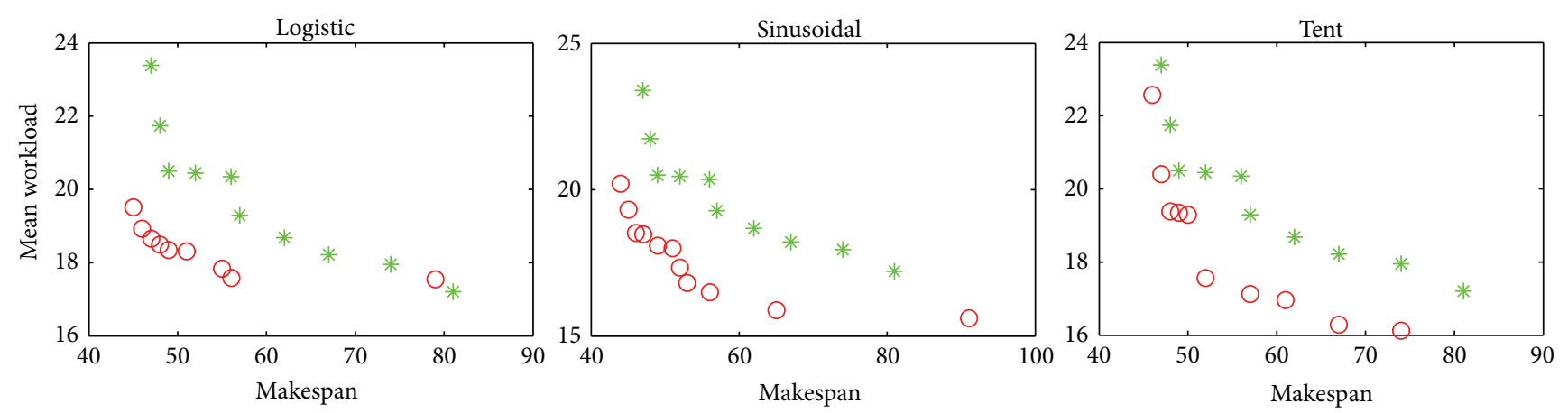

* MOEA/D

* MOEA/D

* MOEA/D

O CMOEA/D-C7

O CMOEA/D-C8

O CMOEA/D-C9

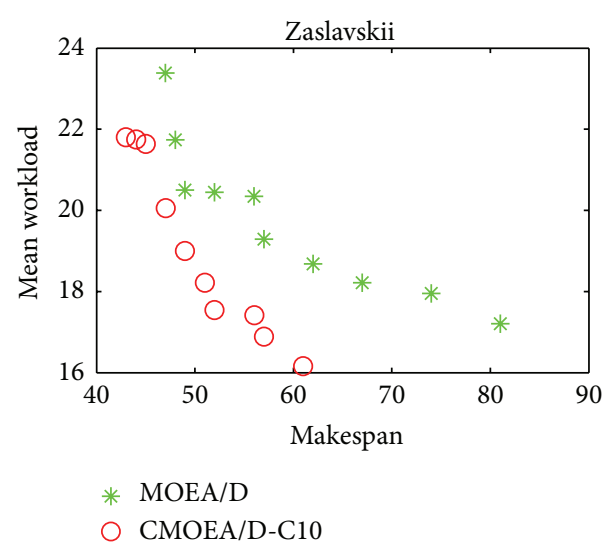

FIgURE 12: Comparison of different chaotic maps for crossover for $40 \times 12$. 

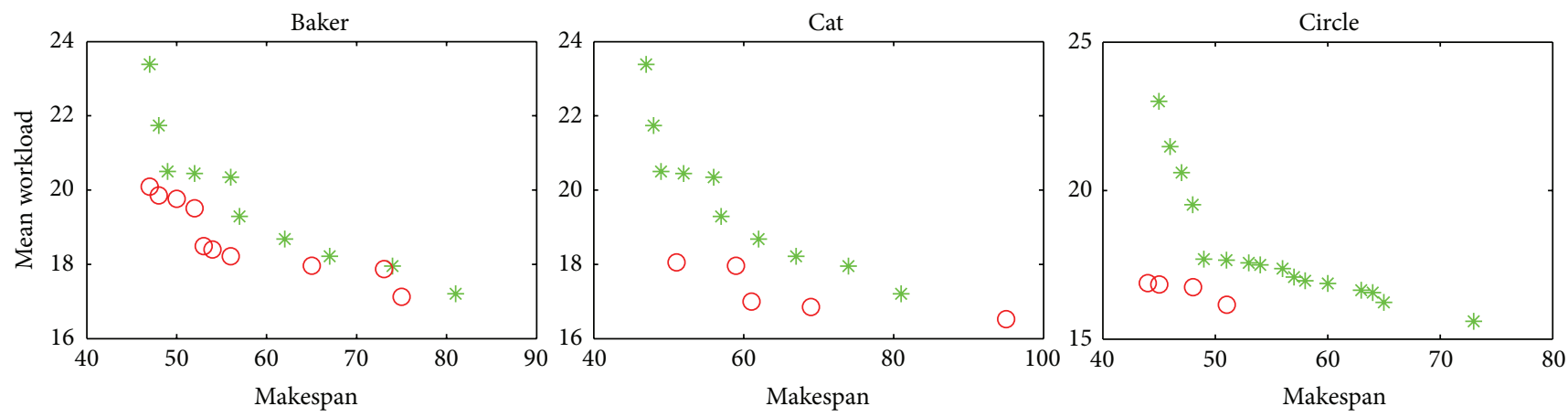

* MOEA/D

O CMOEA/D-I1

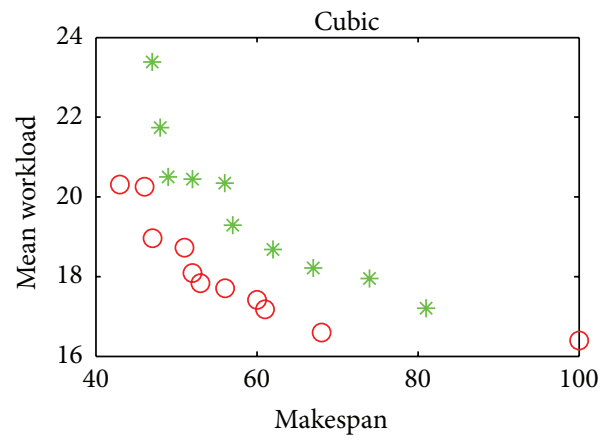

* MOEA/D

O CMOEA/D-I4

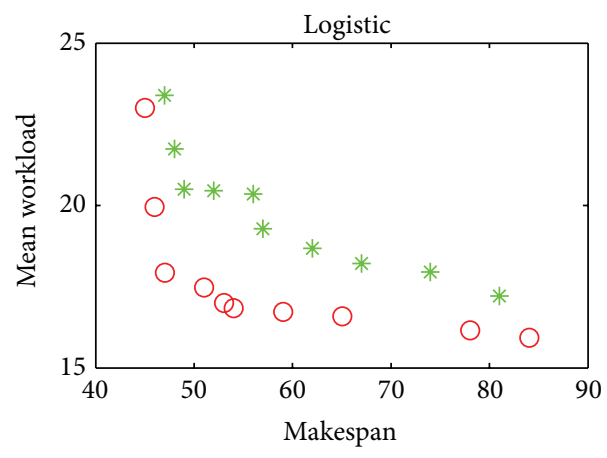

* MOEA/D

O CMOEA/D-I7
* MOEA/D

O CMOEA/D-I2

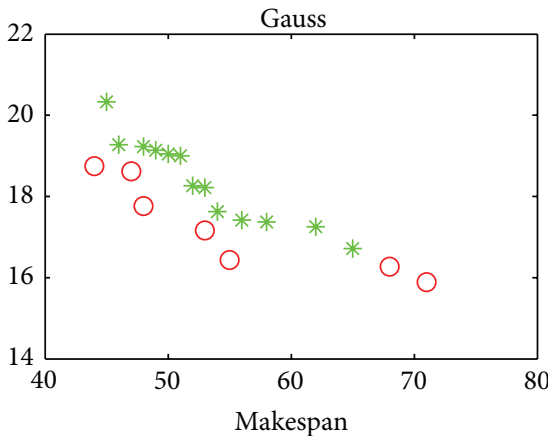

MOEA/D

O CMOEA/D-I3

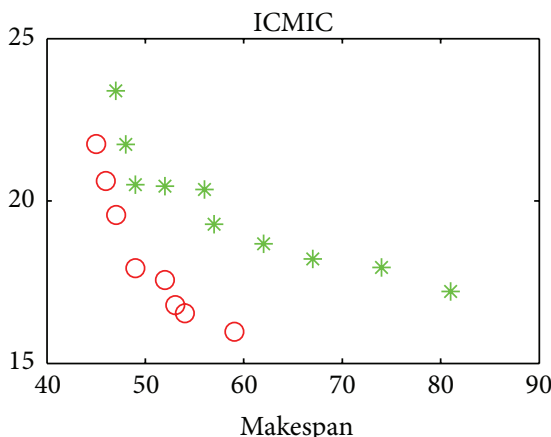

* MOEA/D

O CMOEA/D-I6

O CMOEA/D-I5
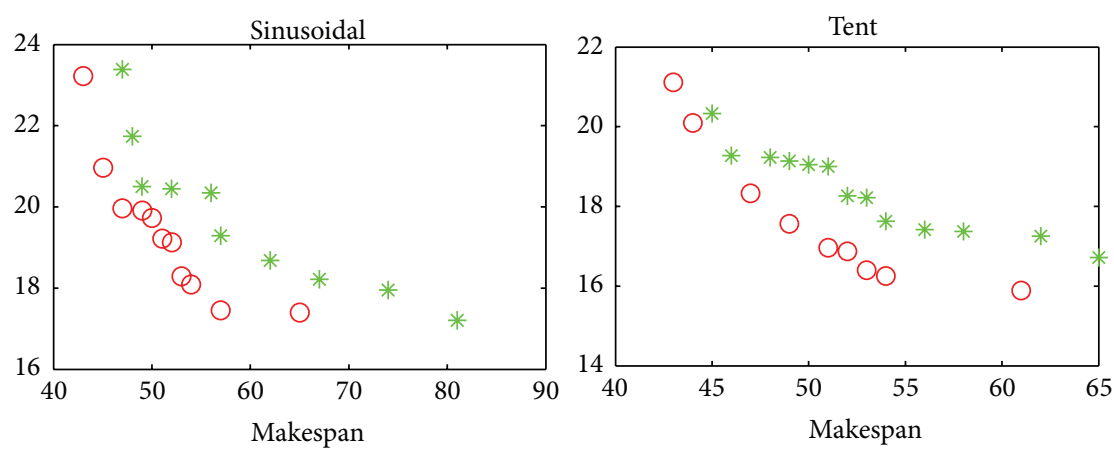

* MOEA/D

O CMOEA/D-I9

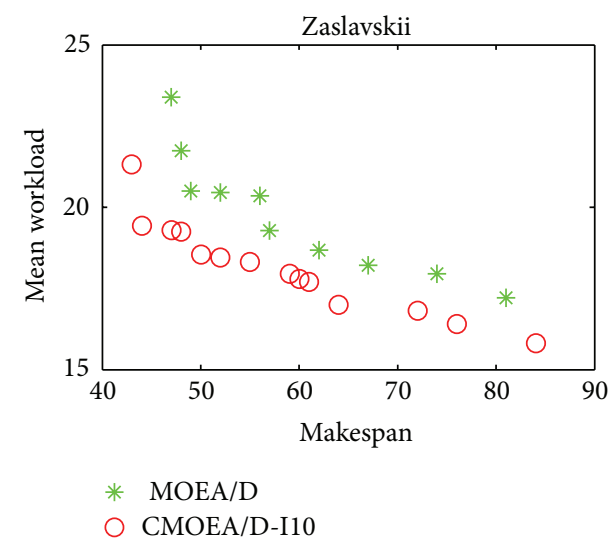

FIgURE 13: Comparison of different chaotic maps for initialization for $40 \times 12$. 

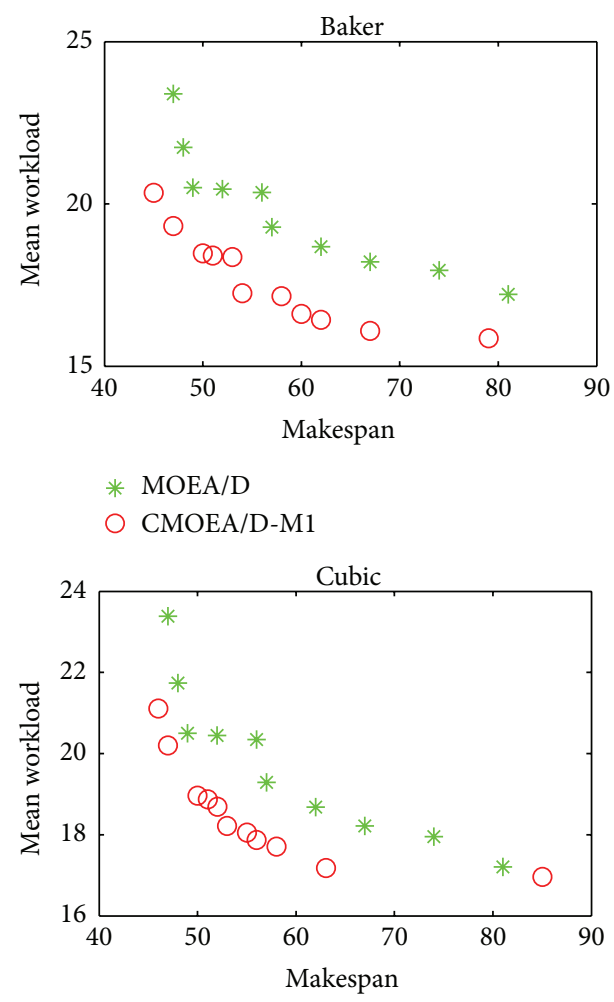

* MOEA/D

O CMOEA/D-M4

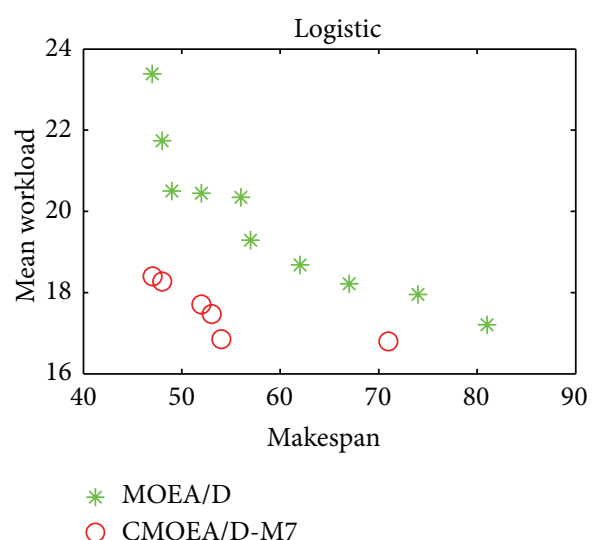

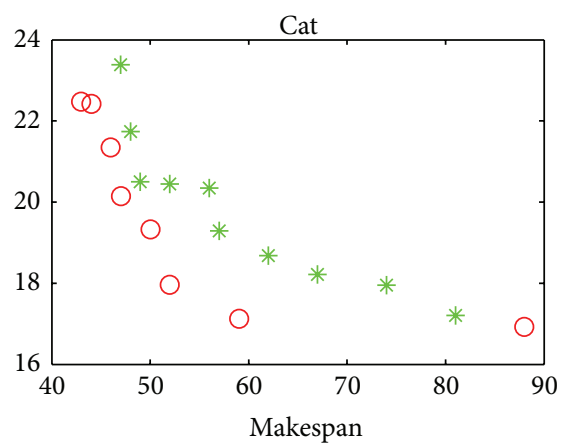
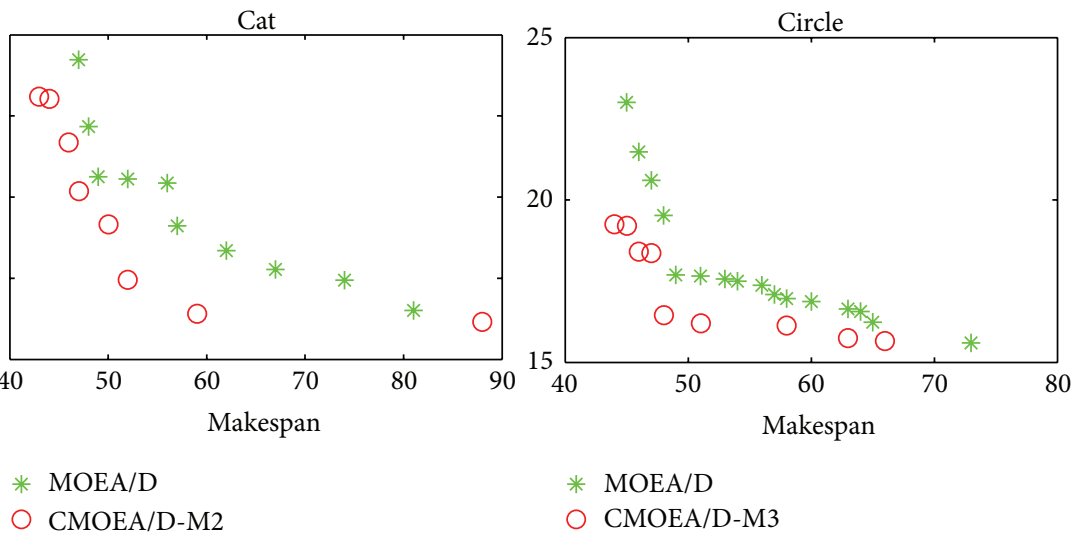

* MOEA/D

O CMOEA/D-M3
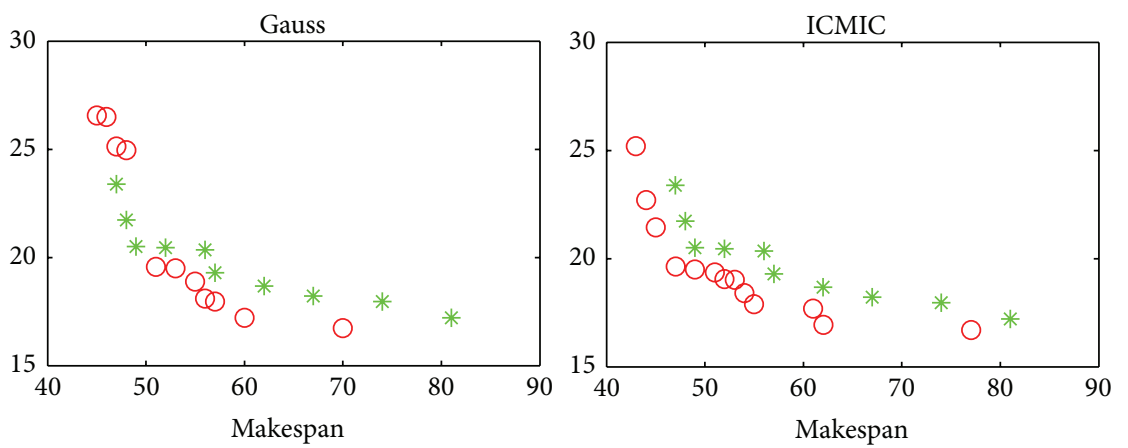

* MOEA/D

O CMOEA/D-M6
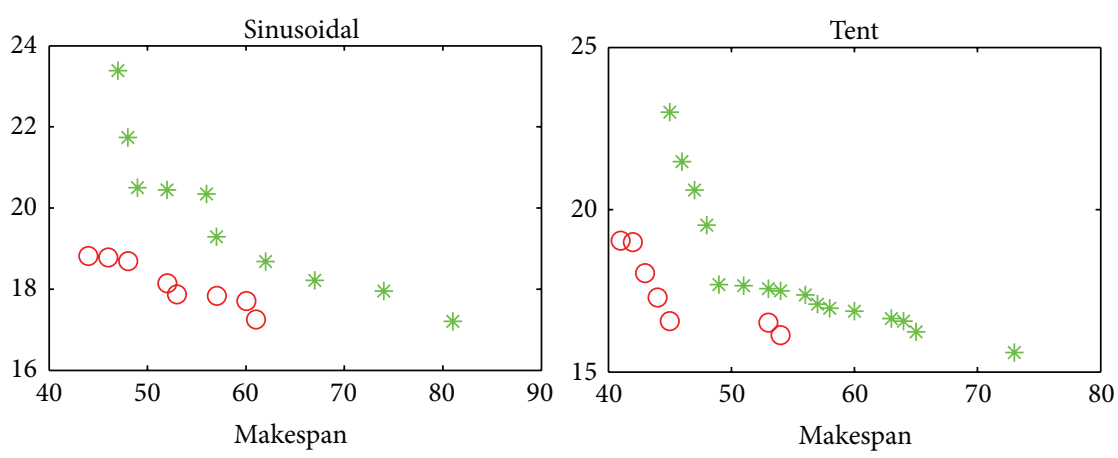

* MOEA/D

○ CMOEA/D-M8

* MOEA/D

O CMOEA/D-M9

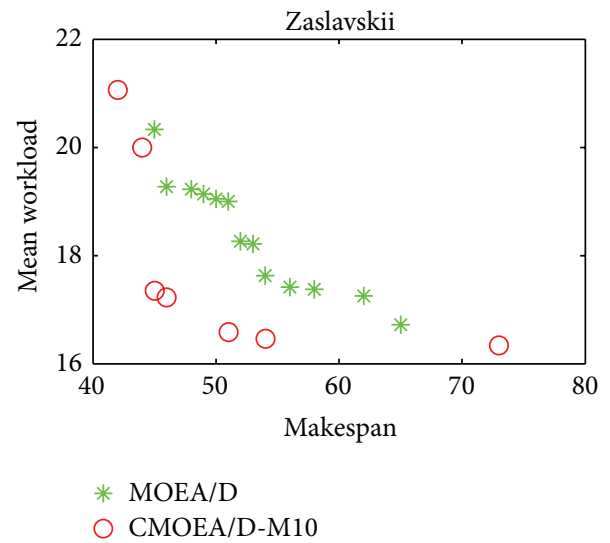

FIgURE 14: Comparison of different chaotic maps for mutation for $40 \times 12$. 


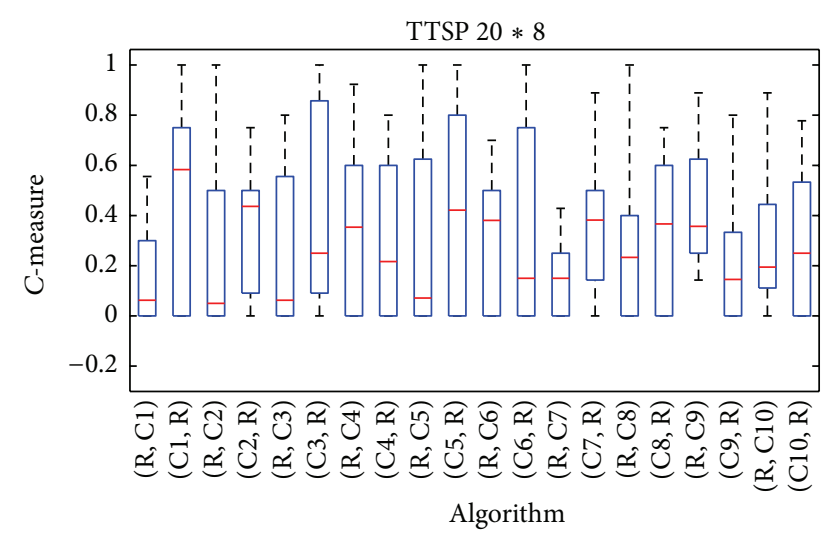

FIgURE 15: The boxplots of $C$ for chaotic maps embedded in crossover.

Here, Leb denotes the Lebesgue measure. Algorithms with larger values of $\mathrm{HV}$ are desirable.

(2) Coverage Metric $C$ (see [11]). The metric $C$ can be used to compare the performances of the two-solution sets. Assume $A$ and $B$ are two sets of nondominated solutions. $C(A, B)$ represents the proportion of points in set $B$ dominated over $A$ in the total points in set $B$. Consider

$$
C(A, B)=\frac{\mid\{x \in B \mid \exists y \in A: y \text { dominates } x\} \mid}{|B|} .
$$

The value $C(A, B)=1$ means that all of the solutions in $B$ are dominated by solutions in $A$, while $C(A, B)=0$ means that no solution in $B$ is dominated by a solution in $A$. Note that both the $C(A, B)$ and $C(B, A)$ have to be considered for comprehensive dominated information for comparing the different set obtained from different algorithm, because $C(A, B) \neq 1-C(B, A)$.

6.4.2. Experiment Results. The average values of performance metrics HV and $C$ of 10 independent runs for both the small and the large scale TTSPs are in Tables 7 and 8, respectively. The symbol is similar to the above mentioned role. In all of the cases, the best performances are denoted in bold.

As shown in Tables 7 and 8, most of the combinations of chaotic maps with MOEA/D have a positive effect for both the small scale and the large scale instances. However, the larger the scale is, the weaker the chaos effect is.

In most cases, the best performance in Table 7 is consistent with that in Table 8. It means the chaotic maps in the specific location have better convergence and comprehensive performances simultaneously. In fact, metric $\mathrm{HV}$ and metric $C$ are different aspects to evaluate the algorithm. Therefore, some inconsistences exist also. Here, we represent the statistical results in an intuitive way. If both the convergence and the comprehensive performances of the algorithm with chaotic maps are better than the original algorithm, the value is replaced by "++." When the situation is opposite, blank is used to replace the corresponding value. Table 9 shows the results.

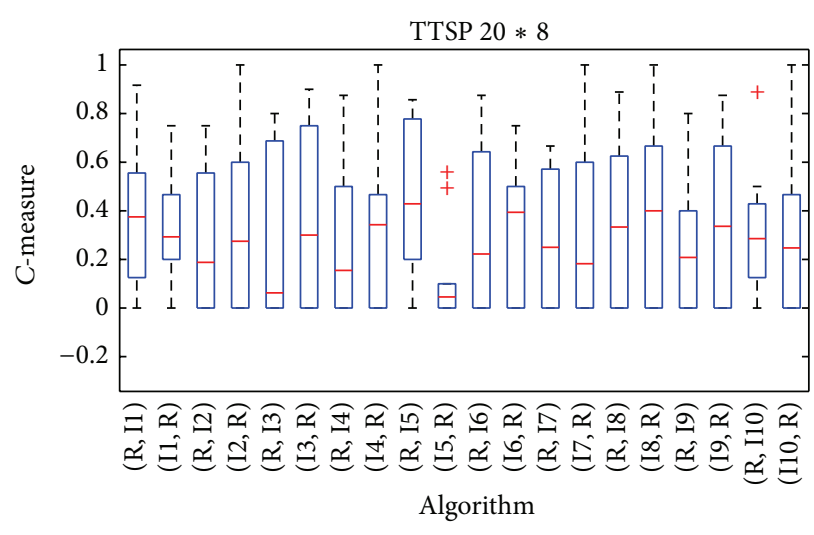

Figure 16: The boxplots of $C$ for chaotic maps embedded in initialization.

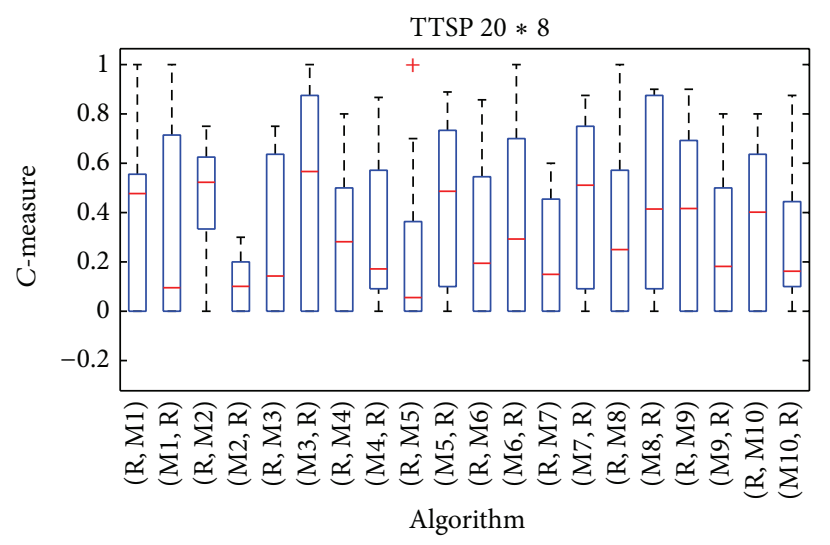

FIgURE 17: The boxplots of $C$ for chaotic maps embedded in mutation.

The results show that circle map and ICMIC map in all phases especially in crossover operator have the best performance. Cubic map and logistic map in mutation operator, Gauss map in crossover operator and mutation operator, sinusoidal map in crossover operator and initial population, baker's map in crossover operator, and Zaslavskii map in initial population have a better effect. In addition, cat map in initial population and mutation operator also has a little bit of effect.

In order to show the above results in an intuitive way, the boxplots of the performance metric $C$ are also adopted to illustrate the same conclusion. Here, we use the boxplots for TTSP $20 \times 8$ as an example. The name role is similar to the above mentioned principle. The ten chaotic maps (baker, cat, circle, cubic, Gauss, ICMIC, logistic, sinusoidal, tent, and Zaslavskii) are denoted by $1,2,3, \ldots, 10$ in alphabetical order. In addition, " $R$ " represents the original MOEA/D. " $I$ " represents the phase for initial population. " $C$ " represents the phase for crossover operator. " $M$ " represents the phase for mutation operator. For example, the algorithm for initial population by logistic map is named " $I 7$." Figures 15,16 , and 17 are the boxplots for chaotic maps embedded in crossover, in initialization, and in mutation, separately. 
TABLE 7: The average value of HV.

\begin{tabular}{|c|c|c|c|c|c|c|c|c|c|c|c|c|}
\hline & \multicolumn{6}{|c|}{$6 \times 8$} & \multicolumn{6}{|c|}{$20 \times 8$} \\
\hline & $R$ & C & $R$ & I & $R$ & $M$ & $R$ & C & $R$ & I & $R$ & $M$ \\
\hline Baker & 0.3312 & 0.3555 & 0.3312 & 0.3240 & 0.3312 & 0.3716 & 0.7471 & 0.7724 & 0.7471 & 0.7472 & 0.7471 & 0.7480 \\
\hline Cat & 0.3312 & 0.3361 & 0.3312 & 0.3592 & 0.3312 & 0.3429 & 0.7471 & 0.7460 & 0.7471 & 0.7580 & 0.7471 & 0.7370 \\
\hline Circle & 0.3312 & 0.3514 & 0.3312 & 0.3538 & 0.3312 & 0.3528 & 0.7471 & 0.7502 & 0.7471 & 0.7556 & 0.7471 & 0.7608 \\
\hline Cubic & 0.3312 & 0.3536 & 0.3312 & 0.3305 & 0.3312 & 0.3511 & 0.7471 & 0.7411 & 0.7471 & 0.7448 & 0.7471 & 0.7595 \\
\hline Gauss & 0.3312 & 0.3470 & 0.3312 & 0.3324 & 0.3312 & 0.3568 & 0.7471 & 0.7538 & 0.7471 & 0.7294 & 0.7471 & 0.7548 \\
\hline ICMIC & 0.3312 & 0.3618 & 0.3312 & 0.3416 & 0.3312 & 0.3672 & 0.7471 & 0.7557 & 0.7471 & 0.7465 & 0.7471 & 0.7488 \\
\hline Logistic & 0.3312 & 0.3450 & 0.3312 & 0.3302 & 0.3312 & 0.3394 & 0.7471 & 0.7579 & 0.7471 & 0.7560 & 0.7471 & 0.7539 \\
\hline Sinusoidal & 0.3312 & 0.3514 & 0.3312 & 0.3373 & 0.3312 & 0.3386 & 0.7471 & 0.7540 & 0.7471 & 0.7483 & 0.7471 & 0.7547 \\
\hline Tent & 0.3312 & 0.3566 & 0.3312 & 0.3318 & 0.3312 & 0.3416 & 0.7471 & 0.7442 & 0.7471 & 0.7486 & 0.7471 & 0.7448 \\
\hline \multirow[t]{3}{*}{ Zaslavskii } & 0.3312 & 0.3276 & 0.3312 & 0.3376 & 0.3312 & 0.3434 & 0.7471 & 0.7466 & 0.7471 & 0.7562 & 0.7471 & 0.7433 \\
\hline & \multicolumn{6}{|c|}{$30 \times 12$} & \multicolumn{6}{|c|}{$40 \times 12$} \\
\hline & $R$ & C & $R$ & I & $R$ & $M$ & $R$ & C & $R$ & I & $R$ & $M$ \\
\hline Baker & 0.5326 & 0.4976 & 0.5326 & 0.5515 & 0.5326 & 0.5398 & 0.7588 & 0.9734 & 0.7588 & 0.7168 & 0.7588 & 0.7395 \\
\hline Cat & 0.5326 & 0.5283 & 0.5326 & 0.5484 & 0.5326 & 0.5708 & 0.7588 & 0.7777 & 0.7588 & 0.7289 & 0.7588 & 0.8604 \\
\hline Circle & 0.5326 & 0.5350 & 0.5326 & 0.5160 & 0.5326 & 0.5357 & 0.7588 & 0.8077 & 0.7588 & 0.7250 & 0.7588 & 0.7538 \\
\hline Cubic & 0.5326 & 0.5164 & 0.5326 & 0.5314 & 0.5326 & 0.5195 & 0.7588 & 0.7576 & 0.7588 & 0.7551 & 0.7588 & 0.7357 \\
\hline Gauss & 0.5326 & 0.5167 & 0.5326 & 0.5194 & 0.5326 & 0.5176 & 0.7588 & 0.7513 & 0.7588 & 0.7726 & 0.7588 & 0.7353 \\
\hline ICMIC & 0.5326 & 0.5428 & 0.5326 & 0.5105 & 0.5326 & 0.5475 & 0.7588 & 0.8214 & 0.7588 & 0.7370 & 0.7588 & 0.7569 \\
\hline Logistic & 0.5326 & 0.5261 & 0.5326 & 0.5458 & 0.5326 & 0.5155 & 0.7588 & 0.7360 & 0.7588 & 0.7064 & 0.7588 & 0.7796 \\
\hline Sinusoidal & 0.5326 & 0.5152 & 0.5326 & 0.5201 & 0.5326 & 0.5181 & 0.7588 & 0.7904 & 0.7588 & 0.6875 & 0.7588 & 0.7440 \\
\hline Tent & 0.5326 & 0.5312 & 0.5326 & 0.5380 & 0.5326 & 0.5187 & 0.7588 & 0.7600 & 0.7588 & 0.7144 & 0.7588 & 0.7881 \\
\hline Zaslavskii & 0.5326 & 0.4911 & 0.5326 & 0.5028 & 0.5326 & 0.5150 & 0.7588 & 0.7506 & 0.7588 & 0.8685 & 0.7588 & 0.8432 \\
\hline
\end{tabular}

TABLE 8: The average value of $C$.

\begin{tabular}{|c|c|c|c|c|c|c|c|c|c|c|c|c|}
\hline & \multicolumn{6}{|c|}{$6 \times 8$} & \multicolumn{6}{|c|}{$20 \times 8$} \\
\hline & $(R, C)$ & $(C, R)$ & $(R, I)$ & $(I, R)$ & $(R, M)$ & $(M, R)$ & $(R, C)$ & $(C, R)$ & $(R, I)$ & $(I, R)$ & $(R, M)$ & $(M, R)$ \\
\hline Baker & 0.0400 & 0.0583 & 0.0400 & 0.0000 & 0.0000 & 0.1000 & 0.1753 & 0.5005 & 0.3741 & 0.3233 & 0.3815 & 0.3572 \\
\hline Cat & 0.0000 & 0.0000 & 0.0000 & 0.0667 & 0.0000 & 0.0250 & 0.2800 & 0.3847 & 0.2873 & 0.3517 & 0.4660 & 0.1102 \\
\hline Circle & 0.0500 & 0.0833 & 0.0367 & 0.0917 & 0.0250 & 0.0500 & 0.2496 & 0.3992 & 0.2713 & 0.3785 & 0.2672 & 0.4786 \\
\hline Cubic & 0.0900 & 0.0833 & 0.0250 & 0.0583 & 0.0750 & 0.0833 & 0.3770 & 0.3111 & 0.2801 & 0.3596 & 0.2964 & 0.3158 \\
\hline Gauss & 0.0250 & 0.1083 & 0.0250 & 0.0250 & 0.0250 & 0.0833 & 0.2982 & 0.4293 & 0.4517 & 0.1346 & 0.2475 & 0.4288 \\
\hline ICMIC & 0.0000 & 0.0583 & 0.0450 & 0.0833 & 0.0250 & 0.0833 & 0.3017 & 0.3641 & 0.3097 & 0.3347 & 0.2637 & 0.3743 \\
\hline Logistic & 0.0900 & 0.0583 & 0.0250 & 0.0583 & 0.0000 & 0.0250 & 0.1479 & 0.4037 & 0.2874 & 0.3465 & 0.2338 & 0.4438 \\
\hline Sinusoidal & 0.0250 & 0.0500 & 0.0500 & 0.0583 & 0.0500 & 0.0250 & 0.2652 & 0.3502 & 0.3466 & 0.4186 & 0.3333 & 0.3158 \\
\hline Tent & 0.0000 & 0.0750 & 0.0500 & 0.0000 & 0.0250 & 0.0833 & 0.4352 & 0.2210 & 0.2500 & 0.3592 & 0.3911 & 0.2708 \\
\hline \multirow[t]{3}{*}{ Zaslavskii } & 0.0000 & 0.0000 & 0.0000 & 0.0333 & 0.0250 & 0.0250 & 0.2804 & 0.2911 & 0.3236 & 0.2762 & 0.3490 & 0.2720 \\
\hline & \multicolumn{6}{|c|}{$30 \times 12$} & \multicolumn{6}{|c|}{$40 \times 12$} \\
\hline & $(R, C)$ & $(C, R)$ & $(R, I)$ & $(I, R)$ & $(R, M)$ & $(M, R)$ & $(R, C)$ & $(C, R)$ & $(R, I)$ & $(I, R)$ & $(R, M)$ & $(M, R)$ \\
\hline Baker & 0.5861 & 0.2929 & 0.5208 & 0.2383 & 0.4730 & 0.4039 & 0.2847 & 0.4455 & 0.6541 & 0.1514 & 0.4676 & 0.3046 \\
\hline Cat & 0.4136 & 0.3699 & 0.4242 & 0.3134 & 0.3993 & 0.4263 & 0.5515 & 0.2536 & 0.5098 & 0.1996 & 0.4573 & 0.2652 \\
\hline Circle & 0.2573 & 0.4552 & 0.4339 & 0.3152 & 0.3287 & 0.3220 & 0.4333 & 0.4729 & 0.4678 & 0.3517 & 0.3883 & 0.3801 \\
\hline Cubic & 0.4172 & 0.2880 & 0.4579 & 0.3487 & 0.3989 & 0.3229 & 0.4228 & 0.2989 & 0.5586 & 0.2629 & 0.4272 & 0.3358 \\
\hline Gauss & 0.4304 & 0.3084 & 0.4930 & 0.2412 & 0.4560 & 0.3533 & 0.3788 & 0.3788 & 0.5212 & 0.4023 & 0.3629 & 0.3899 \\
\hline ICMIC & 0.3204 & 0.5210 & 0.5540 & 0.2483 & 0.4886 & 0.3886 & 0.3237 & 0.4530 & 0.6220 & 0.2232 & 0.3899 & 0.3902 \\
\hline Logistic & 0.3935 & 0.3342 & 0.5051 & 0.2571 & 0.4668 & 0.3420 & 0.4870 & 0.3450 & 0.5793 & 0.2007 & 0.3341 & 0.4106 \\
\hline Sinusoidal & 0.4075 & 0.4172 & 0.5211 & 0.2960 & 0.4670 & 0.3727 & 0.4508 & 0.3314 & 0.7251 & 0.1100 & 0.4354 & 0.4003 \\
\hline Tent & 0.3002 & 0.3803 & 0.4465 & 0.3732 & 0.4913 & 0.2751 & 0.4500 & 0.2876 & 0.5798 & 0.2495 & 0.3538 & 0.4387 \\
\hline Zaslavskii & 0.6872 & 0.1425 & 0.5136 & 0.3129 & 0.5040 & 0.3512 & 0.3968 & 0.3680 & 0.2626 & 0.5035 & 0.4932 & 0.3487 \\
\hline
\end{tabular}


TABLE 9: The visualized result.

\begin{tabular}{|c|c|c|c|c|c|c|c|c|c|c|c|c|}
\hline & \multicolumn{3}{|c|}{$6 \times 8$} & \multicolumn{3}{|c|}{$20 \times 8$} & \multicolumn{3}{|c|}{$30 \times 12$} & \multicolumn{3}{|c|}{$40 \times 12$} \\
\hline & C & $I$ & $M$ & $C$ & $I$ & $M$ & $C$ & $I$ & $M$ & $C$ & $I$ & $M$ \\
\hline Baker & ++ & & ++ & ++ & & & & & & ++ & & \\
\hline Cat & & ++ & ++ & & ++ & & & & ++ & & & \\
\hline Circle & ++ & ++ & ++ & ++ & ++ & ++ & ++ & & & ++ & & \\
\hline Cubic & & & ++ & & & ++ & & & & & & \\
\hline Gauss & ++ & & ++ & ++ & & ++ & & & & & & \\
\hline ICMIC & ++ & ++ & ++ & ++ & & ++ & ++ & & & ++ & & \\
\hline Logistic & & & ++ & ++ & ++ & ++ & & & & & & ++ \\
\hline Sinusoidal & ++ & ++ & & ++ & ++ & & & & & & & \\
\hline Tent & ++ & & ++ & & ++ & & & & & & & ++ \\
\hline Zaslavskii & & ++ & & & & & & & & & ++ & \\
\hline
\end{tabular}

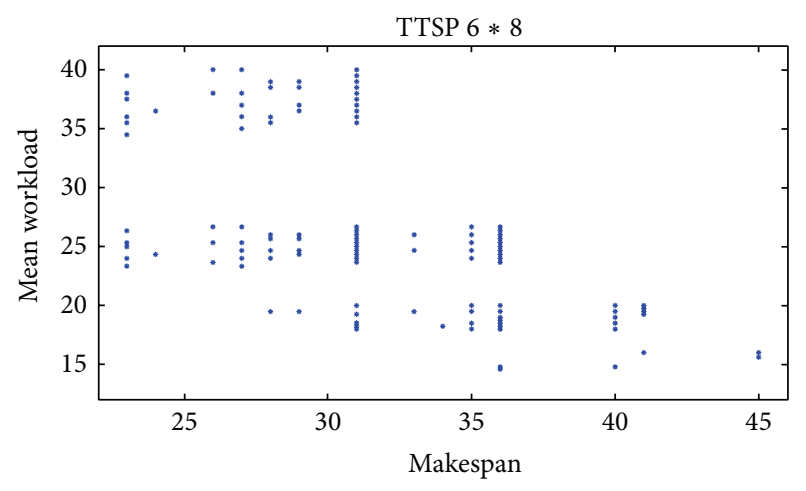

* Solutions

Figure 18: Exhaustive result for TTSP $6 \times 8$.

Overall, chaotic maps for crossover and mutation operators are helpful for preventing the solutions from trapping in the local optima and have significant improvement on the evolutionary algorithms based on the decomposition for solving the TTSP. Circle map and ICMIC map have the best performance in ten maps especially. Cubic map, logistic map, Gauss map, and sinusoidal map have better contribution in solving those TTSPs.

6.4.3. Result Analysis. We discuss and explore the reason for these conclusions based on the above results. We focus on the distribution of solutions of the TTSP.

We calculate the feasible solutions of a small scale TTSP $6 \times 8$ using the method of enumeration that cannot be used in large scale TTSPs. The result is shown in Figure 18. The solutions for the true Pareto front are $[(23,70 / 3) ;(28,19.5) ;(31,18) ;(36,14.6)]$ out of 103,680 solutions in objective space. We can find that the TTSP has nonuniform distribution, and many local optima exist among all the solutions of the TTSP.

The chaotic map has the nature to avoid becoming trapped in local optima. The TTSP has many local optima. All the experiments illustrate the fact that using chaotic maps embedded with the evolutionary algorithm can help the TTSP to obtain good solutions. In addition, the process of crossover and mutation is important for jumping out of local optima. The experiments also validate this fact.

Furthermore, chaotic maps have a superior effect on escaping from local optima, but not all of them are effective. We want to find the relationship from the distribution. The distribution of every chaotic map is shown in Figure 1. Some chaotic maps, like circle map, cubic map, and ICMIC map, are relatively nonuniformly distributed. It is very similar to the distribution of the optimal solution of the TTSP. The above experiments indicate that these chaotic maps have a positive effect on TTSP. Some chaotic maps, like cat map, have uniform distribution. The experiments show that they cannot obtain good effect for solving the TTSP in most situations. It is natural that the effect of chaotic maps is floating under different circumstance, because of the ergodicity and stochasticity of chaotic maps. However, the similarity degree of the distribution between the chaotic maps and the problem is a very essential factor for the application of chaotic maps.

6.5. Experiment 4: Comparison of CMOEA/D and VNM. Referring to Table 9, together with the data in Tables 7 and 8 , we select a few variants of CMOEA/D to compare with VNM. VNM has been proved to be more suitable to solve the TTSP than other methods such as chaotic NSGA-II (CNSGA) [5]. Therefore, a comparison of CMOEA/D and VNM is carried out to illustrate the performance of our algorithm.

We take TTSP $20 \times 8$ and $40 \times 12$ as representative test problems. For TTSP $20 \times 8$, we select the three variants of CMOEA/D. They are CMOEA/D-C1 with baker's map in crossover operator, CMOEA/D-I9 with tent map in the initial population, and CMOEA/D-M3 with circle map in the mutation operator, respectively. For TTSP $40 \times 12$, the three variants of CMOEA/D are CMOEA/D-C1 with baker's map in crossover operator, CMOEA/D-I10 with Zaslavskii map in the initial population, and CMOEA/D-M9 with tent map in the mutation operator, respectively. The results of the performance metrics $\mathrm{HV}$ and $\mathrm{C}$ of 10 independent runs are in Tables 10 and 11, separately. The best results obtained from VNM and three variants of CMOEA/D for different instances are shown in Figures 19 and 20. "V" represents the VNM, and the symbol in the table is similar to the above mentioned role. 

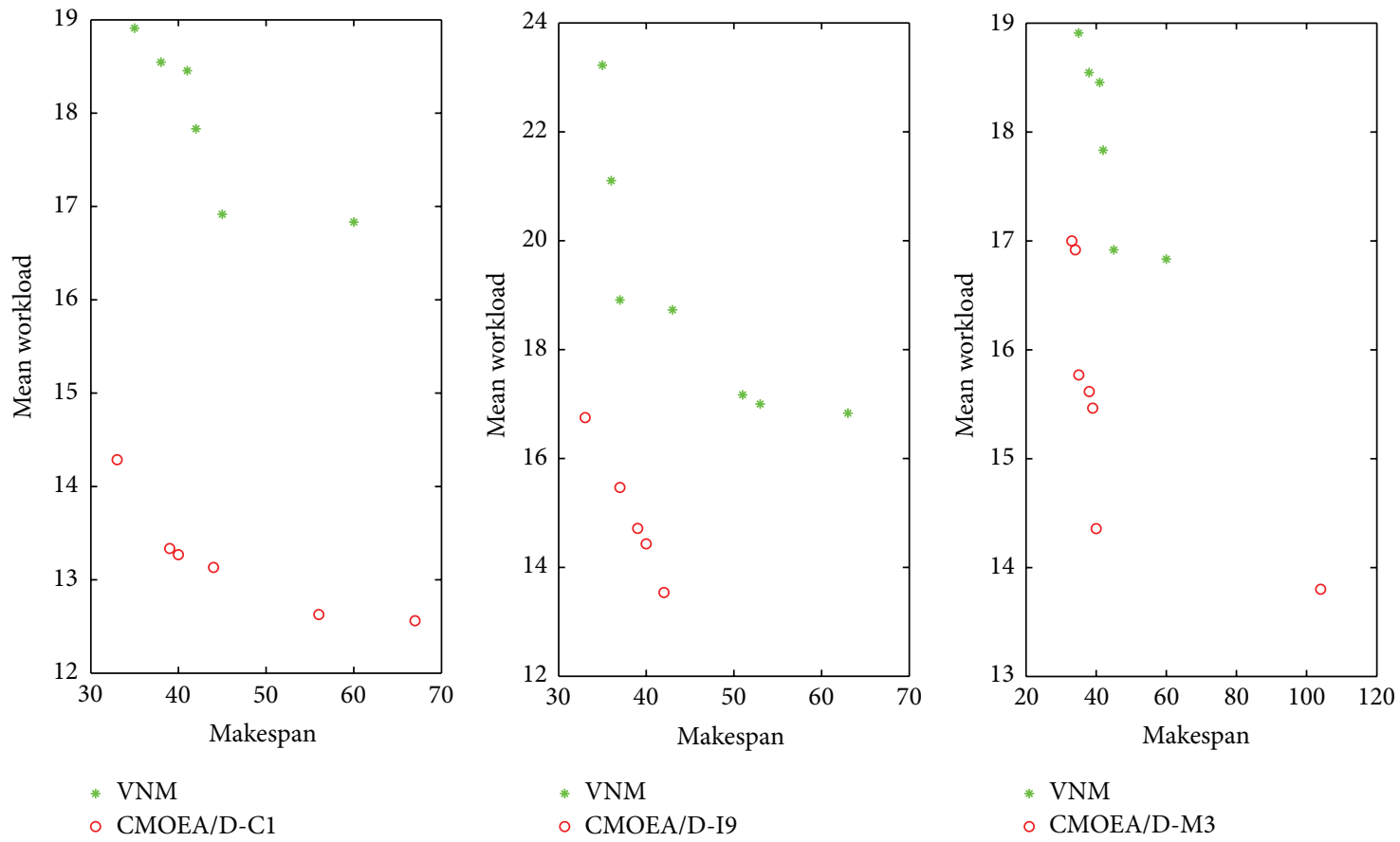

FIGURE 19: Comparison of VNM and three variants of CMOEA/D for $20 \times 8$.
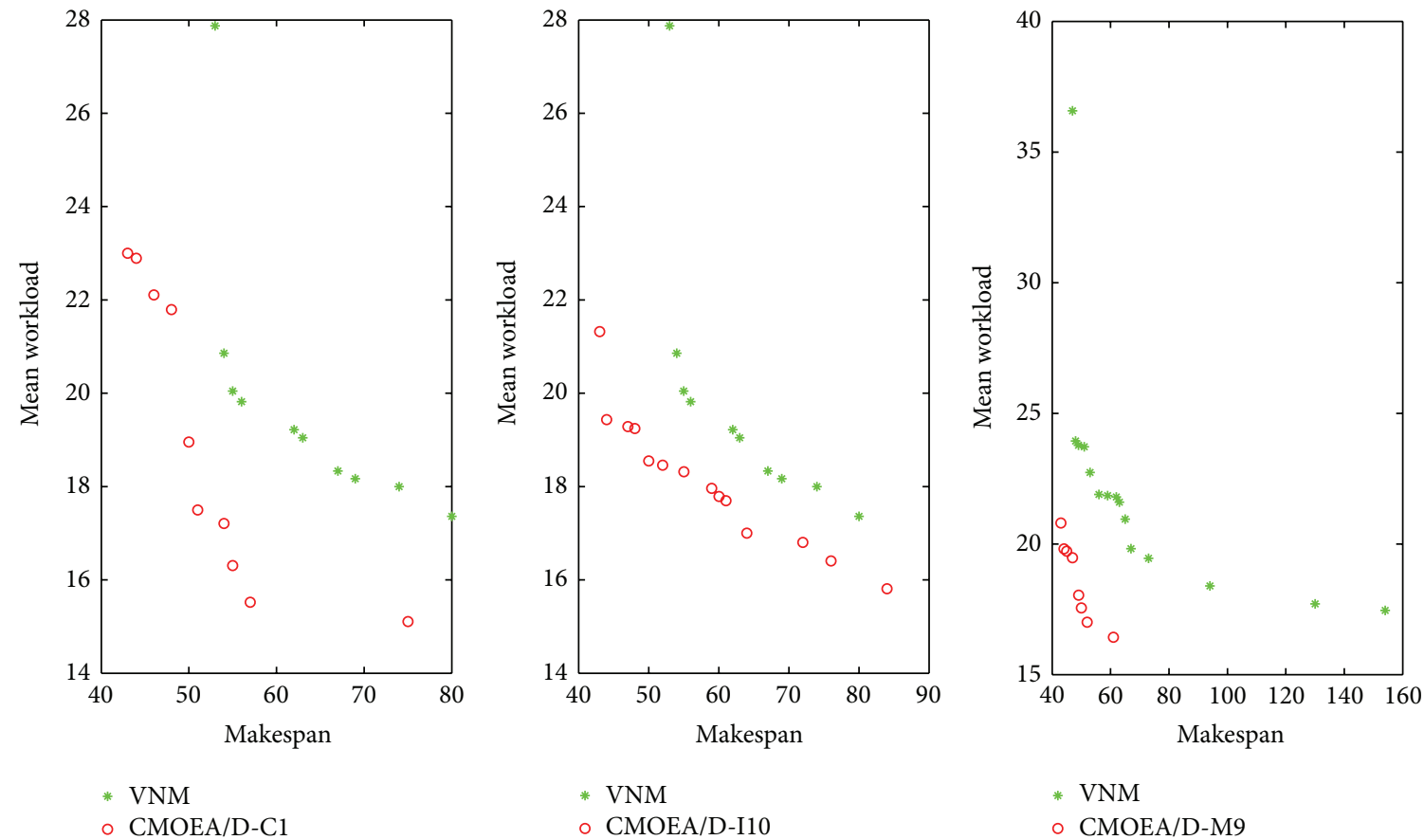

FIgURE 20: Comparison of VNM and three variants of CMOEA/D for $40 \times 12$.

It shows that the solutions obtained by the CMOEA/D dominate most of the solutions obtained by the VNM in the above figures. The values of $C$ in Table 11 indicate that CMOEA/D has good convergence. The results in Table 10 also show that the solutions obtained by CMOEA/D are of higher comprehensive performance. Therefore, the CMOEA/D has the best performance completely.

A short summary can be obtained according to the above experiments and analyses. The improving encoding method is effective for solving the TTSP. In addition, the 
TABLE 10: The value of HV.

\begin{tabular}{|c|c|c|c|c|c|c|c|c|c|c|c|c|}
\hline & \multicolumn{6}{|c|}{$20 \times 8$} & \multicolumn{6}{|c|}{$40 \times 12$} \\
\hline & V & C & V & $I$ & V & $M$ & V & C & V & $I$ & V & $M$ \\
\hline 1 & 0.4067 & 0.4928 & 0.4067 & 0.4582 & 0.4067 & 0.4642 & 0.4016 & 0.4718 & 0.4016 & 0.4576 & 0.4016 & 0.4189 \\
\hline 2 & 0.4018 & 0.4844 & 0.4018 & 0.4756 & 0.4018 & 0.4746 & 0.4148 & 0.4511 & 0.4148 & 0.4449 & 0.4148 & 0.4521 \\
\hline 3 & 0.4552 & 0.4776 & 0.4552 & 0.4582 & 0.4552 & 0.4706 & 0.4729 & 0.4729 & 0.4729 & 0.4937 & 0.4729 & 0.4222 \\
\hline 4 & 0.4258 & 0.4907 & 0.4258 & 0.4789 & 0.4258 & 0.4643 & 0.3881 & 0.4853 & 0.3881 & 0.4521 & 0.3881 & 0.4286 \\
\hline 5 & 0.4283 & 0.5055 & 0.4283 & 0.4607 & 0.4283 & 0.4755 & 0.4010 & 0.4598 & 0.4010 & 0.4674 & 0.4010 & 0.4666 \\
\hline 6 & 0.4492 & 0.4690 & 0.4492 & 0.4738 & 0.4492 & 0.4889 & 0.4155 & 0.4389 & 0.4155 & 0.4496 & 0.4155 & 0.4354 \\
\hline 7 & 0.4560 & 0.4540 & 0.4560 & 0.4612 & 0.4560 & 0.4871 & 0.3900 & 0.4521 & 0.3900 & 0.4688 & 0.3900 & 0.4578 \\
\hline 8 & 0.4306 & 0.4765 & 0.4306 & 0.4688 & 0.4306 & 0.4789 & 0.3968 & 0.4426 & 0.3968 & 0.4703 & 0.3968 & 0.4750 \\
\hline 9 & 0.4460 & 0.4872 & 0.4460 & 0.4632 & 0.4460 & 0.4740 & 0.4235 & 0.4585 & 0.4235 & 0.4500 & 0.4235 & 0.4468 \\
\hline 10 & 0.4458 & 0.4897 & 0.4458 & 0.4803 & 0.4458 & 0.4767 & 0.4002 & 0.4406 & 0.4002 & 0.4465 & 0.4002 & 0.4537 \\
\hline Average & 0.4345 & 0.4827 & 0.4345 & 0.4679 & 0.4345 & 0.4755 & 0.4104 & 0.4574 & 0.4104 & 0.4601 & 0.4104 & 0.4457 \\
\hline Times & 1 & 9 & 0 & 10 & 0 & 10 & 0 & 9 & 0 & 10 & 1 & 9 \\
\hline
\end{tabular}

TABLE 11: The value of $C$.

\begin{tabular}{lccccccccccccc}
\hline & \multicolumn{9}{c}{$20 \times 8$} \\
& $(V, C)$ & $(C, V)$ & $(V, I)$ & $(I, V)$ & $(V, M)$ & $(M, V)$ & $(V, C)$ & $(C, V)$ & $(V, I)$ & $(I, V)$ & $(V, M)$ & $(M, V)$ \\
\hline 1 & 0.0000 & 1.0000 & 0.0000 & 0.8333 & 0.0000 & 1.0000 & 0.0000 & 1.0000 & 0.0000 & 1.0000 & 0.0000 & 0.9000 \\
2 & 0.0000 & 1.0000 & 0.0000 & 1.0000 & 0.0000 & 1.0000 & 0.0000 & 1.0000 & 0.0000 & 1.0000 & 0.0000 & 1.0000 \\
3 & 0.0000 & 0.9000 & 0.3750 & 0.5000 & 0.1667 & 0.7000 & 0.0000 & 1.0000 & 0.0000 & 1.0000 & 0.0000 & 1.0000 \\
4 & 0.0000 & 1.0000 & 0.0000 & 1.0000 & 0.0000 & 1.0000 & 0.0000 & 1.0000 & 0.0000 & 1.0000 & 0.0000 & 1.0000 \\
5 & 0.0000 & 1.0000 & 0.0000 & 0.9000 & 0.0000 & 1.0000 & 0.0000 & 1.0000 & 0.0000 & 1.0000 & 0.0000 & 1.0000 \\
6 & 0.0000 & 0.1667 & 0.0833 & 0.1667 & 0.0000 & 1.0000 & 0.0000 & 0.8571 & 0.0000 & 1.0000 & 0.1000 & 0.8571 \\
7 & 0.4444 & 0.0000 & 0.0000 & 0.2000 & 0.0000 & 1.0000 & 0.0000 & 1.0000 & 0.0000 & 1.0000 & 0.0000 & 1.0000 \\
8 & 0.0000 & 1.0000 & 0.0000 & 1.0000 & 0.0000 & 1.0000 & 0.0000 & 1.0000 & 0.0000 & 1.0000 & 0.0000 & 1.0000 \\
9 & 0.0000 & 1.0000 & 0.0000 & 1.0000 & 0.0000 & 1.0000 & 0.0000 & 1.0000 & 0.0000 & 0.7778 & 0.0000 & 0.7778 \\
10 & 0.0000 & 1.0000 & 0.1000 & 0.5714 & 0.0833 & 0.5714 & 0.0000 & 1.0000 & 0.0000 & 1.0000 & 0.0000 & 1.0000 \\
\hline Average & 0.0444 & $\mathbf{0 . 8 0 6 7}$ & 0.0558 & $\mathbf{0 . 7 1 7 1}$ & 0.0250 & $\mathbf{0 . 9 2 7 1}$ & 0.0000 & $\mathbf{0 . 9 8 5 7}$ & 0.0000 & $\mathbf{0 . 9 7 7 8}$ & 0.0100 & $\mathbf{0 . 9 5 3 5}$ \\
Times & 1 & $\mathbf{9}$ & 0 & $\mathbf{1 0}$ & 0 & $\mathbf{1 0}$ & 0 & $\mathbf{1 0}$ & 0 & $\mathbf{1 0}$ & 0 \\
\hline
\end{tabular}

effectiveness of the multiobjective evolutionary algorithm based on decomposition using chaotic maps, which have nonuniform distributions, is illustrated for TTSP. Furthermore, the comparisons of CMOEA/D and VNM indicate that our algorithm has the best performance for solving the TTSP. The fact, the chaotic map is an effective and efficient method for solving the problem with local optima, is illustrated again.

\section{Conclusion}

The TTSP is a complex combinational optimization problem and has many local optima. This paper focuses on the chaotic multiobjective evolutionary algorithm based on decomposition for solving the TTSP. The improving encoding method is proposed to increase the encoding efficiency. Ten chaotic maps are embedded in three phases of MOEA/D to solve the TTSP, and the results show that the proposed algorithm can prevent solutions from falling into local optima. The performance metrics $\mathrm{HV}$ and $C$ are used to analyze the algorithms with chaotic maps. In the experimental results, almost all chaotic maps have good effects on improving the performance of evolutionary algorithms to solve the TTSP.
The CMOEA/D approaches using the circle and ICMIC maps in all phases have best performance and are very suitable for solving the TTSP. A comparison of CMOEA/D and VNM is carried out to test the performance of our algorithm, and the results also show that the solutions obtained by CMOEA/D are of higher comprehensive performance. Our work gives guidance on choosing chaotic maps and phases for the TTSP. Future work will focus on more chaotic maps embedded in other algorithms for different kinds of problems and discover the reasons for their special properties.

\section{Conflict of Interests}

The authors declare that there is no conflict of interests regarding the publication of this paper.

\section{Acknowledgments}

The authors would like to thank the anonymous reviewers for their helpful comments in improving their paper. This research is supported by the National Natural Science Foundation of China under Grant no. 61101153. 


\section{References}

[1] H. Lu, R. Niu, J. Liu, and Z. Zhu, "A chaotic non-dominated sorting genetic algorithm for the multi-objective automatic test task scheduling problem," Applied Soft Computing Journal, vol. 13, 2013.

[2] R. Xia, M. Q. Xiao, and J. J. Cheng, "Parallel TPS design and application based on software architecture, components and patterns," in IEEE Autotestcon, pp. 234-240, Baltimore, Md, USA, 2007.

[3] D. Zhou, P. Qi, and T. Liu, "An optimizing algorithm for resources allocation in parallel test," in Proceedings of the IEEE International Conference on Control and Automation (ICCA '09), pp. 1997-2002, Christchurch, New Zealand, December 2009.

[4] H. Lu, J. Liu, R. Y. Niu, and Z. Zhu, "Fitness distance analysis for parallel genetic algorithm in the test task scheduling problem," Soft Computing, 2013.

[5] H. Lu, Z. Zhu, X. T. Wang, and L. J. Yin, "A variable neighborhood MOEAD for multi-objective test task scheduling problem," Mathematical Problems in Engineering, vol. 2014, Article ID 423621, 14 pages, 2014.

[6] H. Lu, X. Chen, and J. Liu, "Parallel test task scheduling with constraints based on hybrid particle swarm optimization and taboo search," Chinese Journal of Electronics, vol. 21, no. 4, pp. 615-618, 2012.

[7] D. Donald, S. Roman, Z. Ivan, P. Michal, and B. D. Magdalena, "Utilising the chaos-induced discrete self organising migrating algorithm to solve the lot-streaming flowshop scheduling problem with setup time," Soft Computing, vol. 18, no. 4, pp. 669-681, 2014.

[8] M. Gavrilova and K. Ahmadian, "On-demand chaotic neural network for broadcast scheduling problem," Journal of Supercomputing, vol. 59, no. 2, pp. 811-829, 2012.

[9] H. M. Jiang, C. K. Kwong, W. H. Ip, and Z. Q. Chen, "Chaosbased fuzzy regression approach to modeling customer satisfaction for product design," IEEE Transactions on Fuzzy Systems, vol. 21, no. 5, pp. 926-936, 2013.

[10] M. Sun, L. Zhao, W. Cao, Y. Xu, X. Dai, and X. Wang, "Novel hysteretic noisy chaotic neural network for broadcast scheduling problems in packet radio networks," IEEE Transactions on Neural Networks, vol. 21, no. 9, pp. 1422-1433, 2010.

[11] E. Zitzler and L. Thiele, "Multiobjective evolutionary algorithms: a comparative case study and the strength Pareto approach," IEEE Transactions on Evolutionary Computation, vol. 3, no. 4, pp. 257-271, 1999.

[12] C. Cheng, W. Wang, D. Xu, and K. W. Chau, "Optimizing hydropower reservoir operation using hybrid genetic algorithm and chaos," Water Resources Management, vol. 22, no. 7, pp. 895909, 2008.

[13] D. Liu and Y. D. Cao, "CGA: chaotic genetic algorithm for fuzzy job scheduling in grid environment," in Computational Intelligence and Security, vol. 4456 of Lecture Notes in Computer Science, pp. 133-143, 2007.

[14] M. R. Singh and S. S. Mahapatra, "A swarm optimization approach for flexible flow shop scheduling with multiprocessor tasks," The International Journal of Advanced Manufacturing Technology, vol. 62, no. 1-4, pp. 267-277, 2012.

[15] J. M. Bahi, C. Guyeux, A. Makhoul, and C. Pham, "Secure scheduling of wireless video sensor nodes for surveillance applications," in Ad Hoc Networks, vol. 89 of Lecture Notes of the Institute for Computer Sciences, Social Informatics and Telecommunications Engineering, pp. 1-15, 2012.

[16] A. Q. Yu and X. S. Gu, "An improved transiently chaotic neural network approach for identical parallel machine scheduling," in Advances in Cognitive Neurodynamics ICCN 2007, pp. 909-913, 2007.

[17] T. Niknam, M. R. Narimani, J. Aghaei, and R. AzizipanahAbarghooee, "Improved particle swarm optimisation for multiobjective optimal power flow considering the cost, loss, emission and voltage stability index," IET Generation, Transmission and Distribution, vol. 6, no. 6, pp. 515-527, 2012.

[18] R. Zhou, C. M. Ye, and H. M. Ma, "Model research of multi-objective and resource-constrained project scheduling problem," in Proceedings of the 19th International Conference on Industrial Engineering and Engineering Anagement, pp. 9911001, 2013.

[19] Z. M. Fang, "A quantum immune algorithm for multiobjective parallel machine scheduling," in Advances in Swarm Intelligence Lecture Notes in Computer Science, vol. 6145, pp. 321-327, 2010.

[20] Q. Zhang and H. Li, "MOEA/D: a multiobjective evolutionary algorithm based on decomposition," IEEE Transactions on Evolutionary Computation, vol. 11, no. 6, pp. 712-731, 2007.

[21] H. Peitgen, H. Jurgens, and D. Saupe, Chaos and Fractals, Springer, Berlin, Germany, 1992. 


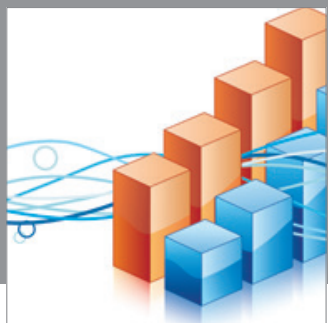

Advances in

Operations Research

mansans

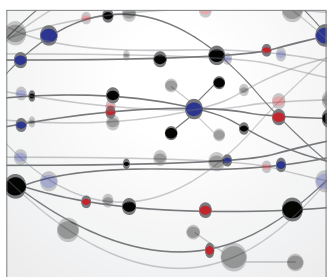

The Scientific World Journal
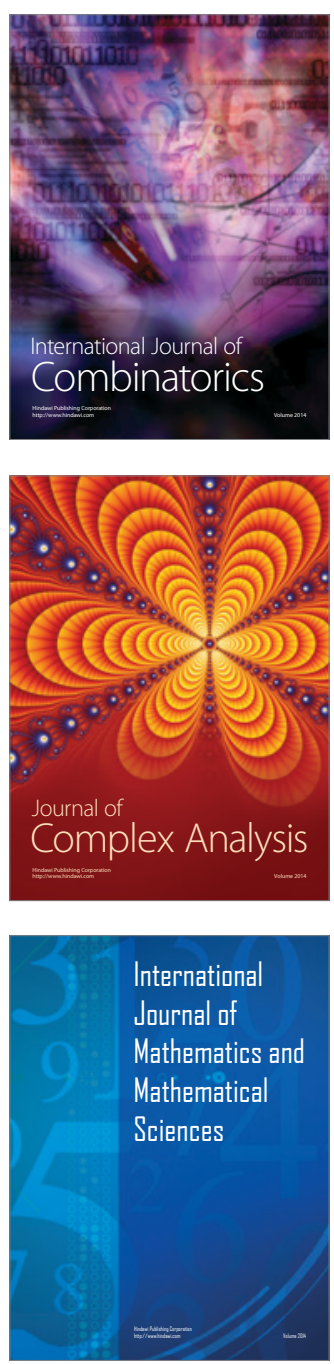
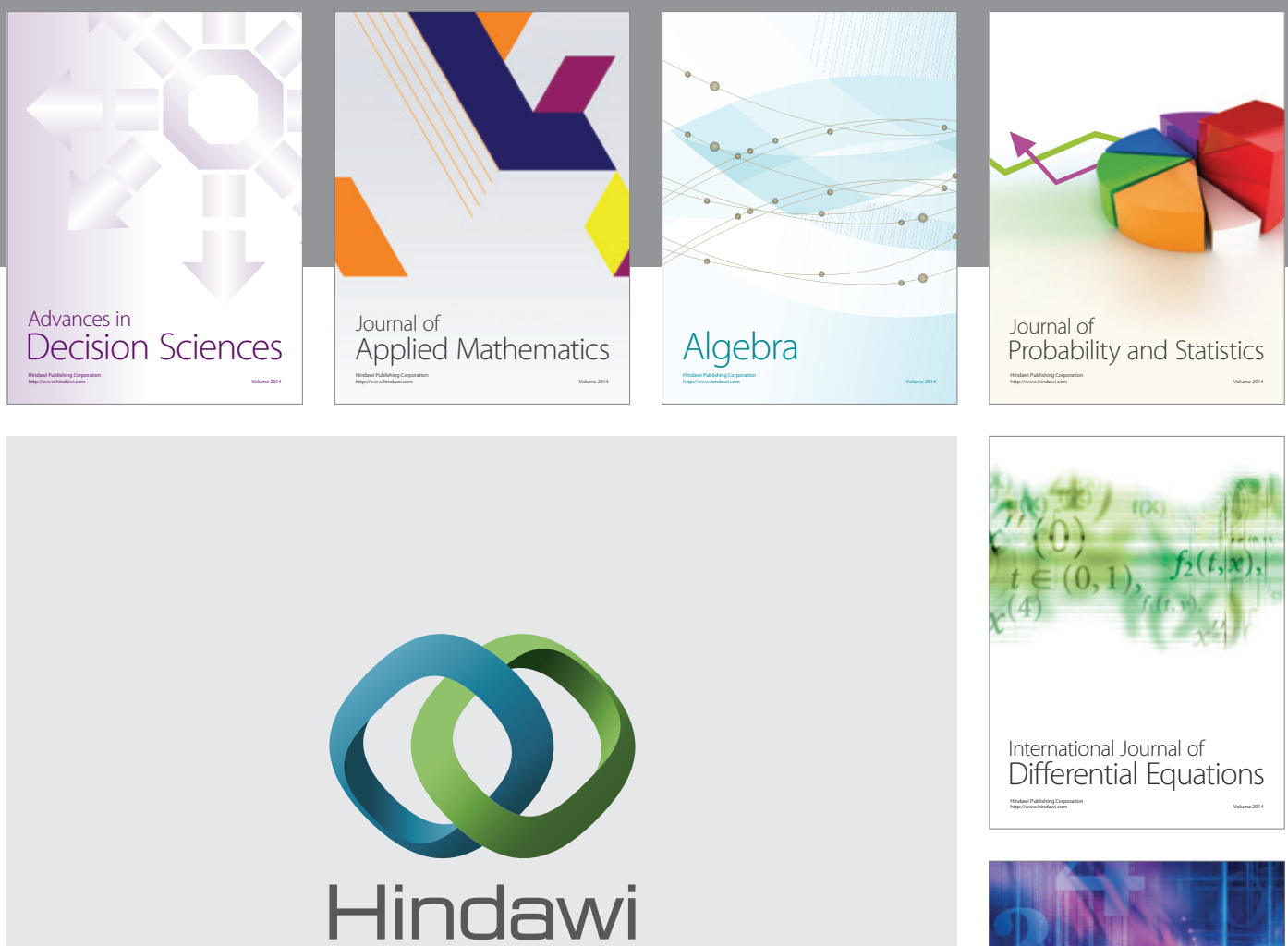

Submit your manuscripts at http://www.hindawi.com
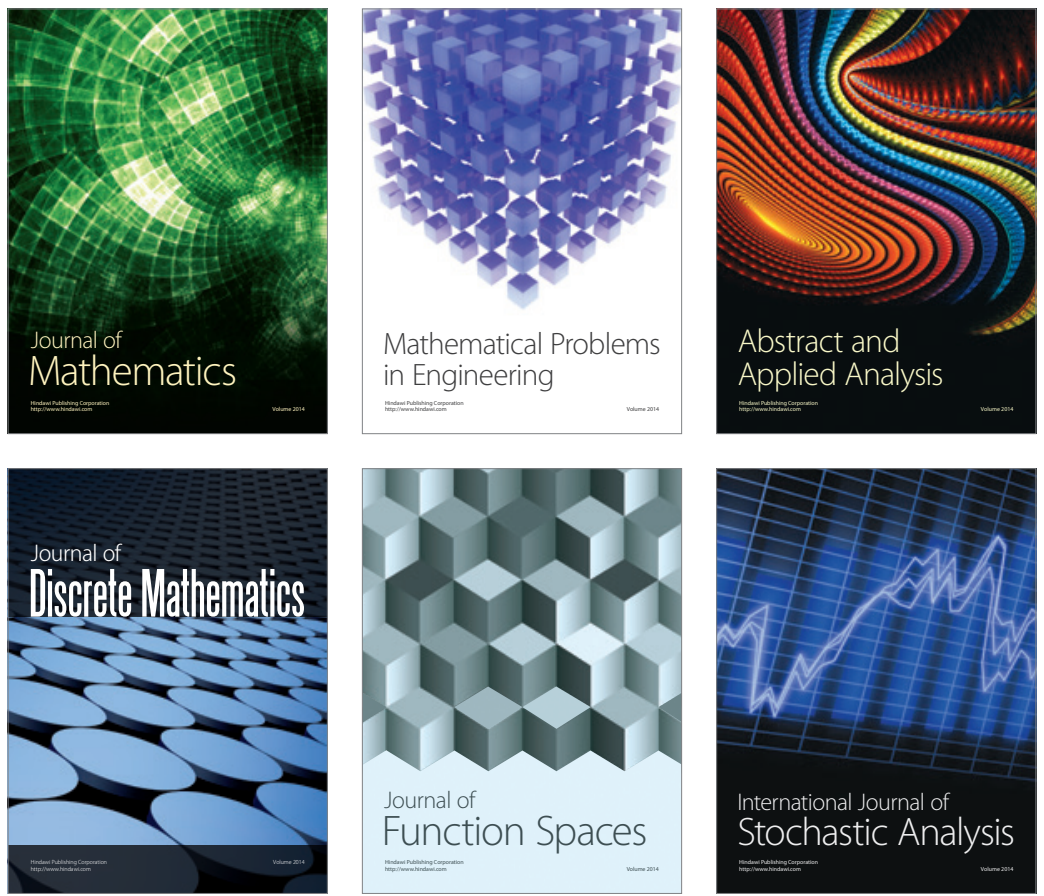

Journal of

Function Spaces

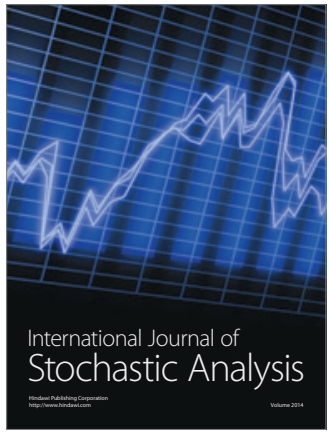

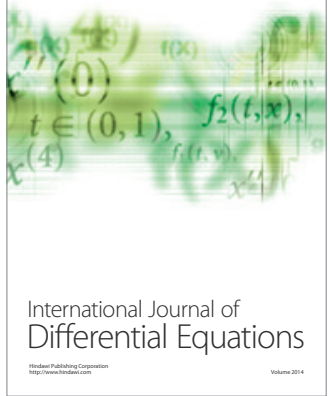
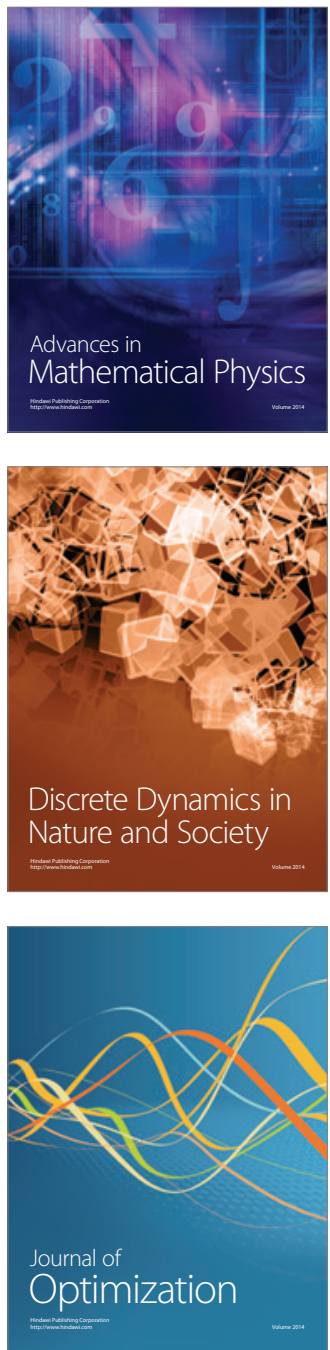